\title{
Asset pricing with endogenous state-dependent risk aversion
}

\author{
Rachida Ouysse**
}

January 24, 2020

\begin{abstract}
We present an economy where aggregate risk aversion is stochastic and state-dependent in response to information about the wider economy. A factor model is used to link aggregate risk aversion to the business cycle and to handle high-dimensionality of the information about the economy. Our estimated aggregate risk aversion is counter-cyclical and varies with news about economic booms and busts. We find new evidence of volatility clustering of risk aversion around recessions. In addition to the price of consumption risk associated with consumption risk, time variation in risk aversion introduces risk preferences as a new component of the risk premium.
\end{abstract}

Key words and phrases: Consumption-based capital asset pricing model; time-varying risk aversion; GMM estimation; Euler equations; mispricing; Counter-cyclicality.

${ }^{* *}$ Corresponding author. School of Economics, The University of New South Wales, Sydney, 2052 Australia. Email: rouysse@unsw.edu.au. 


\section{Introduction}

In financial economics, consumption-based asset pricing models have been among the leading multiperiod general equilibrium asset pricing models for the past three decades. See Breeden et al. (2014) for a review of the origins and development of the consumptionbased capital asset pricing (CCAPM) theory. The CCAPM links asset pricing with macroeconomic risk and states that the expected excess return on any risky asset should be proportional to the asset's "consumption beta." Securities with a higher sensitivity of returns to movements in real consumption spending have more systematic risk and should have proportionately higher excess returns.

The first two decades of CCAPM tests rejected the model's implied first order conditions in its representative agent formulation with time-separable power utility and constant relative risk aversion (Hansen and Singleton (1982a), Mehra and Prescott (1985a)); and the model proved dissapointing in explaining the cross-section of average excess returns (Mankiw and Shapiro (1986), Breeden et al. (1989), Campbell (1996), Cochrane (1996)).

The weak early empirical support for the CCAPM is contrasted with a very strong theory in support of the consumption-based model. 'As a measure of systematic risk, an asset's covariance with the marginal utility of consumption achieves a degree of theoretical purity that is unmatched by other asset pricing models,' Lettau and Ludvigson (2001), page 1239. This disconnect between the theory and empirical evidence motivated researchers to improve the theoretical modeling of CCAPM and its empirical implementation.

In this paper, we formulate and empirically test a consumption-based capital asset pricing model in which aggregate risk aversion is time-varying. We model the relationship between risk aversion and the business cycle through a dynamic multinomial model.

In the literature, attempts to resolve the equity premium and the risk-free rate 
puzzles have taken many directions, some of which emphasize stochastic prices of consumption risk or distorted beliefs. The view of time-varying aggregate preferences was made popular in the literature with the inception of habit formation models Abel (1990), Constantinides (1990), Heaton (1995)). In Campbell and Cochrane s (1999b) habit formation model, the representative agent's risk aversion varies with differences between consumption and the agent's habit formed through past consumption. In habit formation model, the local curvature of the utility function is inversely related to the surplus consumption ratio; therefore, fluctuating risk aversion is counter-cyclical. The investors' risk tolerance is expected to be low during recessions and in times of a sustained decline of prices, and high during exapansions. The relative risk aversion is therefore related with 'recession risk'state variables that are correlated with the business cycle (Maio (2013)).

Time-varying risk aversion also arises in the intertemporal capital asset pricing model (ICAPM) (Merton (1973)), where investors participate in the financial market over a long period of time, thus resulting in situations in which they may wish to hedge against not only shortfalls in consumption but also future changes in investment opportunities.

Brandt and Wang (2003) directly model the time-varying risk aversion by specifying an autoregressive law of motion for the log of relative risk aversion. The innovations in the risk aversion are explicitly related to innovations in consumption growth and inflation. Brunnermeier and Nagel (2008) link time-varying risk aversion to individuals' responses to changes in household wealth.

We formulate and empirically evaluate the implications of a consumption-based capital asset pricing model where risk aversion is time-varying, not only as a response to news about aggregate consumption as in the habit formation model, but also to news about a wide range of key economic indicators. This paper preserves the simple framework of the time-separable risk aversion utility function without using habit formation. Our framework replaces the traditional fixed risk aversion (FRA) with a time-varying 
state-dependent risk aversion (SDRA). We address state-dependencies from a somewhat more general perspective. Our model is a reduced form for an economy in which the representative agent reacts to news about the state of the economy. Instead of specifying which leading economic indicators the agents are responding to, we use the principle of maximum random utility to relate the business cycle indicators to a multinomial state variable. The model can be seen as an approximate reduced form for a more elaborate model with nominal frictions or, money preferences, or as simply capturing cyclical time-variations in aggregate preferences. In contrast to habit formation models, which only allow aggregate preferences to change in response to news about aggregate consumption growth, this model can explicitly specify how aggregate preferences vary through time and in response to the business cycle. In addition to news about aggregate consumption, the model can evaluate such effects as the anxiety about excessive inflation documented in Shiller (1997) by measuring the effect of news about inflation on aggregate preferences.

Instead of specifying a law of motion for the aggregate risk aversion parameter (e.g. autoregressive structure as in Brandt and Wang (2003)), this model links the fluctuations in the aggregate preferences to fluctuations in the business cycle. The latter is modeled as a response to news about the aggregate economy. Our approach is more robust as it avoids issues of mispecification in modeling the time-varying preferenaces. In particular, this paper addresses some of the mitigated empirical performances of the representative agent, A power utility of the constant relative risk aversion. First, models with time and state separable utility functions predict that risk premium (the excess return on risky asset over the riskless asset) is affine in the covariance between consumption growth and returns, with consumption risk priced at a constant rate equal to the coefficient of risk aversion. The observed historical correlation between consumption and returns is low, tand the high risk premia found in the data can only be reconciled with implausibly high risk aversion. Second, in these models the elasticity of intertemporal substitution is the reciprocal of risk aversion, thus predicting a large 
risk-free rate of interest to induce high risk aversion. In this paper, we explore the pricing implications of the time-varying aggregate preferences on the cyclical dynamics of term structure of (nominal) interest rates.

In this paper, we follow the line of research that emphasizes stochastic prices of consumption risks, and we relax the constant relative risk aversion assumption of constant curvature, and consider a business cycle-dependent risk aversion. The model implies two sets of conditional moments: one from the Euler equation and the second from the multinomial logit. The estimation is based on generalized method of moments (GMM) of the joint set of implied unconditional moments. This "joint" estimation provides us with a consistent estimator of the subjective discount rate, the average risk aversion, and the risk aversion sensitivities to changes in the business cycle. Brandt and Wang (2003) use a setup that resembles the habit formation model, which models the dynamics of aggregate risk aversion instead of specifying the agent's habit formation.

Many studies ${ }^{1}$ have indicated that GMM estimators may be badly biased and that the associated tests have substantial size distortions. These shortcomings of the asymptotic approximation might be the result of many possible reasons, inter alia, (1) choice of instruments and the number of lags, (2) choice of the weighting matrix, and (3) choice of alternative GMM estimators.

We use the following notation throughout the paper: $\mathbb{E}\left(. \mid \mathfrak{I}_{t}\right)$ is the expectation of the argument conditional on some suitable information set $\mathfrak{I}_{t}$ available at time $t$, $\mathbb{E}\left(\mathcal{Z}_{t}\right)$ is the unconditional expectation of $\mathcal{Z}_{t}, \mathbb{E}_{F}($.$) is the (unconditional) expectation$ under the probability distribution $F$, the indicator function $\operatorname{Ind}(\mathcal{A})$ takes the value 1 if the statement " $\mathcal{A}$ " is correct and 0 otherwise, $A^{-1}$ is the inverse of $A, \iota_{m}$ is a $m$-vector of ones, $I_{m}$ is an $m \times m$ identity matrix, $\operatorname{diag}(A)$ is the vector consisting of the diagonal elements of $A, \operatorname{tr}(A)$ is the sum of the diagonal elements of $A$, the norm of $A$ is $\|A\|=\left(\operatorname{tr}\left(A^{\prime} A\right)\right)^{1 / 2}, A \otimes B$ is the Kronecker product of $A$ and $B$ defined as $A \otimes B=\left[a_{i j} B\right]$ where $A=\left[a_{i j}\right]$, and 'vector' means a column vector.

\footnotetext{
${ }^{1}$ Several of which have been published in the July 1996 special issue of the Journal of Business 85 Economic Statistics.
} 


\section{Intertemporally separable consumption-based capital asset pricing models}

Consumption-based capital asset pricing models use marginal rates of substitution to determine the relative prices of a composite consumption good which is date and eventcontingent. This model class is characterized by a stochastic discount factor process that puts restrictions on the joint process of asset returns and per capita consumption.

In these models, household trading of financial assets is motivated by a desire to smooth consumption both over time and across states at a point in time. The desirability of an asset reflects its ability to smooth consumption. Assets that pay off in future states when the marginal utility of consumption is low are less desirable than those that pay an equivalent amount in future states when consumption levels are low and additional consumption is more valued.

Consider a single-good general equilibrium model with infinite horizon and identical agents. A representative consumer in this economy chooses stochastic consumption and investment so as to maximize the present value of his lifetime utility:

$$
\max _{C_{t}} \mathbb{E}_{0}\left[\sum_{t=0}^{\infty} \beta^{t} U\left(C_{t}\right),\right]
$$

where $\beta$ is a discount factor in the interval $[0,1]$ and $U($.$) is a strictly concave function.$ Suppose that the consumer can trade freely in some $N$ assets with maturity $n_{j}, j=$ $1, . ., N$.

The feasible set of consumption and investment plans must satisfy the sequence of budget constraints:

$$
C_{t}+\sum_{j=1}^{N} P_{j, t} Q_{j, t} \leqslant R_{j t} Q_{j, t-M_{j}}+W_{t}
$$

where $Q_{j t}$ is the quantity of asset $j$ held at the end of period $t . P_{j t}$ is the price of asset $j$ at period $t . R_{j t}$ is the payoff from holding asset $j$. The investor's first-order condition 


$$
U^{\prime}\left(C_{t}\right)=\beta \mathbb{E}_{t}\left[r_{j, t+1} U^{\prime}\left(C_{t+1}\right)\right]
$$

where $R_{j, t+1}$ is the one period simple gross return from holding one unit of stock $j$ and is defined as:

$$
R_{j, t+1}=\frac{P_{j, t+1}+D_{j, t+1}}{P_{j, t}}
$$

where $D_{j, t+1}$ is the dividend yield on stock $j$ from period $t$ to $t+1$.

The left-hand side of Equation (3) is the marginal utility cost of consuming one less real dollar at time $t$. The right-hand side is the expected marginal utility benefit from investing that dollar in asset $i$ at time $t$ and selling out at time $t+1$, and consuming the proceeds. Equation (3) describes the optimum allocation as the investor equates marginal cost and marginal benefit. The equation can be rewritten as,

$$
1=\beta \mathbb{E}_{t}\left[R_{j, t+1} \frac{U^{\prime}\left(C_{t+1}\right)}{U^{\prime}\left(C_{t}\right)}\right]=\mathbb{E}_{t}\left[R_{j, t+1} M_{t+1}\right]
$$

where $M_{t+1}$ is the stochastic discount factor which characterises the intertemporal marginal rate of substitution of the investor. The stochastic discount factor is unique in complete markets where idiosyncratic variations in investors' marginal utilities are eliminated. Equation (4) must hold for every asset incuding the riskless asset. Applying the properties of expectations, the equation can be expressed as

$$
\mathbb{E}_{t}\left[R_{j, t+1}\right]=\frac{1-\operatorname{Cov}_{t}\left[R_{j, t+1}, M_{t+1}\right]}{\mathbb{E}_{t}\left[M_{t+1}\right]}
$$

An asset with a high expected return must have a low covariance with the stochastic discount factor. This asset is risky in that it fails to deliver wealth when it is most valuable to investors because it tends to have low returns when investors have high marginal utility. Investors demand a large risk premium to hold this type of asset.

Equation (5) must hold equally for the riskless asset whose gross return is $R_{f, t+1}$ 
with the exception that the covariance with the risk-free asset and the stochastic discount factor is zero:

$$
\mathbb{E}_{t}\left[R_{f, t+1}\right]=\frac{1}{\mathbb{E}_{t}\left[M_{t+1}\right]}
$$

Combining Equations (56),

$$
\mathbb{E}_{t}\left[R_{j, t+1}\right]=R_{f, t+1}\left(1-\operatorname{Cov}\left[R_{j, t+1}, M_{t+1}\right]\right) .
$$

To understand the implications of Equation (7), assume, as Hansen and Singleton (1983), that the joint conditional distribution of asset returns and the stochastic discount factor is lognormal and homoskedastic. Taking the logarithm of Equation (6) gives,

$$
\log \left(R_{f, t+1}\right)=-\mathbb{E}_{t}\left(\log \left(M_{t+1}\right)\right)-\frac{\sigma_{m}^{2}}{2}
$$

where $\sigma_{m}^{2}$ denotes the unconditional variance of innovations to the stochastic discount factor. Combining the logarithm of Equation (4) and Equation (8), the excess return on a risky asset over the riskless rate obeys

$$
\mathbb{E}_{t}\left[\log \left(R_{j, t+1}\right)-\log \left(R_{f, t+1}\right)\right]+\frac{\sigma_{j}^{2}}{2}=-\sigma_{j m},
$$

where $\sigma_{j}^{2}$ denotes the unconditional variance of $\log$ return innovations and $\sigma_{j m}$ denotes the unconditional covariance of the innovations in the returns and the stochastic discount factor,

$$
\operatorname{Cov}\left(\log \left(R_{j, t+1}\right)-\mathbb{E}_{t} \log \left(R_{j, t+1}\right), \log \left(M_{t+1}\right)-\mathbb{E}_{t} \log \left(M_{t+1}\right)\right) .
$$

The right-hand side of Equation (9) is the log counterpart of Equation (7) and says that the risk premium is determined by the negative covariance of the asset with the 
stochastic discount factor.

\section{Power utility with constant risk aversion}

Assuming that the preferences are of the constant relative risk aversion (CRRA) type defined over aggregate consumption $C_{t}$

$$
U\left(C_{t}\right)=\frac{C_{t}^{1-\gamma}}{1-\gamma}
$$

where $\gamma$ is the coefficient of relative risk aversion. This power utility is scale invariant and risk premia (given constant return distributions) do not change over time. It also allows aggregation of investors with different wealth levels into one representative agent as long as they all have the same power utility. The marginal utility is $U^{\prime}\left(C_{t}\right)=C_{t}^{-\gamma}$, the stochastic discount factor $M_{t+1}=\beta\left(C_{t+1} / C_{t}\right)^{-\gamma}$, and the elasticity of intertemporal substitution is the reciprocal of the coefficient of relative risk aversion.

For the CRRA power utility function, Equation (6) becomes

$$
\log \left(R_{f, t+1}\right)=-\log \beta+\gamma E_{t} \Delta \log \left(C_{t+1}\right)-\frac{\gamma^{2} \delta_{c}^{2}}{2}
$$

In Equation (10), the riskless real rate is linear in expected consumption growth, with slope coefficient equal to the coefficient of relative risk aversion. The risk premium Equation (7) becomes

$$
\mathbb{E}_{t}\left[\log \left(R_{j, t+1}\right)-\log \left(R_{f, t+1}\right)\right]=\gamma \sigma_{i c}-\frac{\sigma_{j}^{2}}{2}
$$

Equation (11) defines one of the pricing implications of the consumption-based capital asset pricing model with power utility of the constant relative risk aversion family. An asset with a high consumption covariance tends to have low returns when consumption is low and when marginal utility of consumption is high. Such an asset is risky and commands a large risk premium. 
Mehra and Prescott (1985a) documented what has since been known as the equity premium puzzle. The puzzle arizes from the fact that the quantitative predictions of the theory (i.e., Equation (11)) are an order of magnitude different from what has been historically documented in these type of preferences. Campbell (2003) presents empirical implications of the pricing equation in (11). His analysis shows that the implied risk aversion coefficients show that the equity premium puzzle is a robust phenomenon in international data. The implied relative risk aversion coefficents are many times larger than the maximum level 10, considered plausible by Mehra and Prescott (1985a), and are negative in cases where the estimated covariance of stock returns with consumption growth is negative. In addition, the paper indicates that the puzzle arises from the smoothness of consumption rather than the low correlation between consumption and returns.

The high premia found in the data can only be replicated by setting risk aversion to implausibly high levels because of the observed low correlation between consumption and equity. However a high coefficient of relative risk aversion creates another puzzle, the risk-free rate puzzle. Equation $(10)$ implies that the unconditional mean risk-free interest rate is

$$
\mathbb{E} \log R_{f, t+1}=-\log \beta+\gamma \mathbb{E}\left[\log \left(\frac{C_{t+1}}{C_{t}}\right)\right]-\frac{\gamma^{2} \sigma_{c}^{2}}{2}
$$

In historical data, the mean average growth rate of consumption $\mathbb{E} \log \left(C_{t+1} / C_{t}\right)$ is positive. If the coefficint of relative risk aversion is high (which is needed for Equation (11) in the data), then the term $-\frac{\gamma^{2} \sigma_{c}^{2}}{2}$ dominates and pushes the riskless rate down. A low riskless rate is possible in equilibrium only if investors have a low or negative rate of time preference. To reconcile the equity premium puzzle with a high relative risk aversion coefficent implies implausible time preference rates (Campbell, 2003). 


\section{State-dependent aggregate risk aversion}

This paper considers a framework with state-dependent relative risk aversion SDRA, a variation of the CRRA which allows for time-varying attitudes towards risk. The risk aversion parameter $\gamma_{t}$ is time/state-dependent,

$$
U\left(C_{t}, \gamma_{t}, t\right)=\beta \frac{1}{1-\gamma_{t}}\left(C_{t}\right)^{1-\gamma_{t}}, \gamma_{t}>t, \forall t
$$

This utility function can be seen as a special case of more general alternatives to the CRRA in the literature. Gordon and St-Amour (2004) considers preferences where the curvature index $\gamma$ is time-varying. They characterize the representative agent's preferences with within-period conditionally iso-elastic utility,

$$
U\left(C_{t}, \gamma_{t}, t\right)=\beta \frac{\Theta}{1-\gamma_{t}}\left(\frac{C_{t}}{\Theta}\right)^{1-\gamma_{t}}, \gamma_{t}>t, \forall t
$$

where $\Theta>0$ is a constant subjective scaling factor. Gordon and St-Amour (2004) refer to this model as state-dependent risk aversion preferences. The utility function is an implicit function of the state of the world with the key assumption that the process underlying $\gamma_{t}$ is exogenous and unaffected by the agent's decisions. The state variables have an impact on equilibrium returns (through production), but the model rules out cases where "... the state could be related to individual decisions, such as past consumption, or accumulated wealth" (Gordon and St-Amour (2004) p. 243).

The utility function (13) is also a special case of Melino and Yang $\mathrm{s}(2003)$ general state-dependent preferences defined by random consumption sequences constructed as,

$$
U_{t}=\left(\left(1-\beta\left(\mathcal{S}_{t}\right)\right) C_{t}^{\rho\left(\mathcal{S}_{t}\right)}+\beta\left(\mathcal{S}_{t}\right)\left[\mathbb{E}_{t}\left(U_{t+1}^{\alpha\left(\mathcal{S}_{t}\right)}\right)\right]^{\rho\left(\mathcal{S}_{t}\right) / \alpha\left(\mathcal{S}_{t}\right)}\right)^{1 / \rho\left(\mathcal{S}_{t}\right)}
$$

where $\beta\left(\mathcal{S}_{t}\right), \alpha\left(\mathcal{S}_{t}\right)$, and $\rho\left(\mathcal{S}_{t}\right)$ are preference parameters that depend on an exogenous state variable $\mathcal{S}_{t}$. Our setting is a special case of (15), in which the CRRA for 'timeless 
gambles' varies with the state $\gamma_{t}=1-\alpha\left(\mathcal{S}_{t}\right)$, but the measure of time preference parameter $\beta\left(\mathcal{S}_{t}\right)=\beta$ is constant. In addition, our preferences assume $\rho\left(\mathcal{S}_{t}\right)=\rho$ which means that the elasticity of temporal substitution (EIS) is constant and equal to $1 /(1-\rho)$.

$$
U_{t}=\left((1-\beta) C_{t}^{\rho}+\beta\left[\mathbb{E}_{t}\left(U_{t+1}^{1-\gamma_{t}}\right)\right]^{\rho /\left(1-\gamma_{t}\right)}\right)^{1 / \rho}
$$

Gordon and St-Amour (2000, 2004) describe how the instantaneous utility function in (14) captures the effects of pro-cyclical and counter-cyclical risk aversion on marginal utility risk. The level of consumption with respect to the pivot level $\Theta$ determines how changes in the curvature index $\gamma_{t}$ - and hence in risk aversion- marginal utility schedule. When consumption is above the pivot level $\left(C_{t}>\Theta\right)$-resulting from a favorable state - pro-cyclical risk aversion implies a decrease in risk aversion, causing a counter-clockwise rotation in the marginal utility schedule. The converse is true for $C_{t}<\Theta$. When $C_{t} \approx \Theta$, fluctuations in the curvature of utility functions have only limited effects on the marginal utility. Our specification is then a special case in which $\Theta=1$. Gordon and St-Amour (2004) estimated a time-varying risk aversion model in which $\left[R_{t}, \Delta \log \left(\frac{C_{t}}{\Theta}\right), \gamma_{t}\right]$ admits a Multivariate Arithmitic Brownian Motion. In their Bayesian estimation of the model parameters, they find that different values of the scaling metric $\Theta$ do not appear to affect their estimates for risk aversion.

Our approach to risk aversion not being an observed series is to assume that the risk aversion parameter $\gamma$ is dependent on the economic conditions through a state variable $\mathcal{S}_{t}$ in the form of a business cycle dummy which depends on a set of variables $\mathbf{w}_{t}$ that defines the state of the economy. The dependence of $\mathcal{S}_{t}$ and the state variables $\mathbf{w}_{t}$ is modeled using a discrete choice model. The resulting model would be expressed in terms of observed data. For example, $\mathcal{S}_{t}$ can be defined as

$$
\mathcal{S}_{t}=1 \text { when the observation corresponds to a contraction; }
$$


$\mathcal{S}_{t}=2$ when the observation corresponds to a contraction and is an actual trough date;

$\mathcal{S}_{t}=3$ when the observation corresponds to an expansion;

$\mathcal{S}_{t}=4$ when the observation corresponds to an expansion and is an actual peak date.

Alternatively, the state variable can also be simplified to having two states $j=0,1$, where $j=0$ when an observation corresponds to a recession and $j=1$ when an obsevation corresponds to an expansion.

Based on the principle of maximum random utility, assume that there exists an unobserved measure of utility in the economy $U_{j}$ associated with state $j$ of the business cycle. Let us assume that the response variable $\mathcal{S}_{t}$ takes value $j$ if $U_{j}=\max \left\{U_{l}, l \in\right.$ $\left\{1, \cdots, \kappa_{\mathcal{S}}\right\}$. Further assume that $U_{j t}=\mathbf{w}_{t}^{\prime} \beta_{j}+\epsilon_{t}$ where $\epsilon_{t}$ has a CDF $F_{\epsilon}($.$) and \mathbf{w}_{t}$ is a vector of $\mathcal{K}$ variables characterizing the state of the economy at time $t$.

In general, $\mathcal{S}_{t}$ charaterizes the business cycle with $\kappa_{\mathcal{S}}+1$ distinct states. We use a baseline category logit model (Agresti $(2012))$ in which each time period $t$ may be classified into one of $\kappa_{\mathcal{S}}$ categories (states), denoted by $\mathcal{S}_{t}=\left(S_{t 1}, S_{t 2}, \cdots, S_{t \kappa_{\mathcal{S}}}\right)$ the multinomial trial for $t$, where $\mathcal{S}_{t j}=1$ when the time period is in category $j$ and $\mathcal{S}_{t j}=0$ otherwise, $t=1, \cdots, T, j=1, \cdots, \kappa_{\mathcal{S}}$. Thus, $\sum_{j=1}^{\kappa_{\mathcal{S}}} \mathcal{S}_{t j}=1$. We assume that $\left(\mathcal{S}_{t}, \mathbf{w}_{t}\right)$ are independently and identically distributed. Let $\pi_{j}\left(\mathbf{w}_{t}\right)=\mathbb{P}_{t}\left(\mathcal{S}_{t}=j \mid \mathbf{w}_{t}\right)=\mathbb{P}_{t}\left(\mathcal{S}_{t j}=\right.$ $\left.1 \mid \mathbf{w}_{t}\right)$ denote the probability that the observation of $S$ belongs to business cycle state $j$, given covariates $\mathbf{w}_{t}$. We assume the relationship between the probability $\pi_{j t}$ and $\mathbf{w}_{t}$ can be modeled as:

$$
\begin{aligned}
\log \left\{\frac{\pi_{j}\left(\mathbf{w}_{t}\right)}{\pi_{\kappa_{\mathcal{S}}}\left(\mathbf{w}_{t}\right)}\right\} & =\mathbf{w}_{t}^{\prime} \alpha_{j}, \alpha_{j}=\left(\alpha_{j 0}, \alpha_{j 1}, \cdots, \alpha_{j \mathcal{K}}\right)^{\prime} \\
\pi_{j}\left(\mathbf{w}_{t}\right) & =\mathbb{P}\left(U_{j t}=\max \left\{U_{l t}, 1 \leq l \leq \kappa_{\mathcal{S}} \mid \mathbf{w}_{t}\right\}\right) \\
& =\pi_{j t},
\end{aligned}
$$


where $\kappa_{\mathcal{S}}$ is set as a reference state for $\mathcal{S}_{t}$.

For the case of a multinomial logit, we have

$$
\begin{aligned}
& \pi_{j t}=\frac{1}{\sum_{l=1}^{\kappa_{\mathcal{S}}-1} \exp \left(\mathbf{w}_{t}^{\prime}\left(\alpha_{l}-\alpha_{j}\right)\right)} \\
& j=1, \ldots, \kappa_{\mathcal{S}} \text { and } \quad \alpha_{0}=\mathbf{0}_{\kappa} .
\end{aligned}
$$

The dependence of the risk aversion on the state of the economy is modeled through the polytomous response variable $\mathcal{S}_{t}$ :

$$
\gamma_{t}=\mathbb{E}_{t-1}\left(\mathcal{S}_{t} \Gamma+\epsilon_{t}\right),
$$

where $\Gamma=\left(\Gamma_{1}, \cdots, \Gamma_{\kappa_{\mathcal{S}}}\right)^{\prime}$, and $\epsilon_{t}$ are innovations that drive time-variations in risk aversion. If we set state 1 as a reference category, we can redefine the parameter $\gamma$ and write

$$
\gamma_{t}=\mathbb{E}_{t-1}\left(\Gamma_{1}+\sum_{j=2}^{\kappa_{\mathcal{S}}} \mathcal{S}_{t j} \Gamma_{j}+\epsilon_{t}\right)
$$

This model postulates that aggregate risk aversion varies not only in response to news about consumption growth (as in the CRRA) but also to news about the state of the economy. The state variable $\mathcal{S}_{t}$ has information about the business cycle and transmits information about macroeconomic activity to the dynamics of the relative risk aversion. The state-dependent risk aversion dynamics in Equation $(17)$ imply that the state-dependent risk aversion $\gamma_{t}$ is determined by the conditional expectation of the state variables $\mathcal{S}_{t}$

$$
\gamma_{t}=\Gamma_{1}+\sum_{j=2}^{\kappa_{\mathcal{S}}} \Gamma_{j} \mathbb{E}\left(\mathcal{S}_{t j} \mid \mathbf{w}_{t}\right) .
$$

From the specification of the risk aversion (17), one can relate the first moment of $\gamma_{t}$ 
to the economic variables $\mathbf{w}_{t}$

$$
\begin{aligned}
\gamma_{t} & =\Gamma_{1}+\sum_{j=2}^{\kappa_{\mathcal{S}}} \Gamma_{j} \mathbb{P}\left(\mathcal{S}_{t j}=1 \mid \mathbf{w}_{t}\right) \\
\mathbb{P}\left(\mathcal{S}_{t j}=1 \mid \mathbf{w}_{t}\right) & =\frac{\exp \left(\mathbf{w}_{t}^{\prime} \alpha_{j}\right)}{1+\sum_{k=1}^{\kappa_{\mathcal{S}}} \exp \left(\mathbf{w}_{t}^{\prime} \alpha_{k}\right)} .
\end{aligned}
$$

This framework therefore implies two systems of equations defined by the unconditional moments of the model. The first system is impled by the pricing equations in the Euler equation (4) of the CAPM moment conditions, and the second - and new - system corresponds to the unconditional moments of the multinomial model (17). The multinomial system has $\kappa$ unknown parameters. Assuming exogeneity of the explanatory state variables and that $\mathbf{w}_{t}$ includes a column of ones, the following moment conditions

$$
\begin{array}{r}
\mathbb{E}\left[\mathcal{S}_{t j}-\mathbb{P}\left(\mathcal{S}_{t j}=1 \mid \mathbf{w}_{t}\right)\right]=0 \forall j=1, \cdots, \kappa_{\mathcal{S}}, \\
\mathbb{E}\left[\left(\mathcal{S}_{t j}-\mathbb{P}\left(\mathcal{S}_{t j}=1 \mid \mathbf{w}_{t}\right)\right) \otimes \mathbf{w}_{t}\right]=\mathbf{0}_{\kappa} \forall j=1, \cdots, \kappa_{\mathcal{S}}
\end{array}
$$

are valid to estimate the parameters $\alpha_{j}, j=1, \cdots, \kappa_{\mathcal{S}}$. This multinomial logit specification of the dynamics of the time-varying relative risk aversion $\gamma_{t}$ completes the description of the aggregate preferences in our setup.

\section{Econometric model and GMM estimation}

Models that are defined in terms of conditional moment restrictions establish that certain parametric functions have zero conditional mean when evaluated at the parameter values. Let $\left\{\mathbf{y}_{t}\right\}_{t=1}^{T}$ be an ergodic and stationary time series vector of endogenous and exogenous random variables. The coordinates of $\mathbf{y}_{t}$ are related by an econometric model which establishes that the true distribution of data satisfies the conditional moment 
restrictions

$$
\mathbb{E}\left[\zeta\left(\mathbf{y}_{t+1}, \Theta_{0}\right) \mid \mathfrak{I}_{t}\right]=0, t=1, \ldots, T-1,
$$

for a unique value of the $\phi$-vector $\Theta_{0} \in \Theta$, where $\Theta \subset \mathbb{R}^{\phi}$ and $\zeta\left(\mathbf{x}_{t+1}, \Theta_{0}\right)$ are an $\ell$-dimensional parametric function. The function $\zeta$ can be understood as the errors measuring the deviation from an equilibrium condition. Suppose that we can form an $T \times \varphi$ matrix $\mathcal{Z}$ with typical row $\mathcal{Z}_{t}$ such that all its elements belong to $\mathfrak{I}_{t}$. The $\varphi$ variables given by the columns of $\mathcal{Z}$ are called instrumental variables, or simply instruments. These instruments are required to be 'predetermined' and are not necessarily 'econometrically' exogenous. That is, current and lagged values of $\mathbf{y}$ are valid instruments.

The generalized methods of moments estimation (GMM) of Hansen and Singleton (1982b) permits direct estimation and inference of the nonlinear rational expectation model defined by the population orthogonality conditions in (27) of the equilibrium stochastic Euler equations. The GMM circumvents the need of a complete, explicit characterization of the environment and does not require strong assumptions about the forcing variables of the equilibrium path. Given the conditional moment restriction (27) and the additional assumption that the constituents of $\zeta\left(\mathbf{y}_{t+1}, \Theta_{0}\right)$ and the variables in $\mathcal{Z}_{t}$ have finite second moments (Hansen and Singleton (1982a)), a family of unconditional population orthogonality conditions

$$
\mathbb{E}\left[g\left(\mathcal{X}_{t}, \Theta_{0}\right)\right]=0,
$$

can be constructed where $\mathcal{X}_{t} \equiv\left(\mathbf{y}_{t+1}, \mathcal{Z}_{t}\right), g_{t}\left(\mathcal{X}_{t}, \Theta_{0}\right)=\zeta\left(\mathbf{y}_{t+1}, \Theta_{0}\right) \otimes \mathcal{Z}_{t}$. The moment restrictions in (25) are also known as the estimating equations.

Let $c_{t+1}$ be the ratio of consumption at time $t+1$ to the consumption at time $t$, and assume for simplicity that assets are held for one period $n_{j}=1$, then for $j=1, \ldots, m$, the Euler equation (4) for the consumer optimization problem can be rewritten as a 
residual form

$$
E_{t}\left[\beta \frac{C_{t+1}^{-\gamma_{t+1}}}{C_{t}^{-\gamma_{t}}} R_{j, t+1}-1\right]=0,
$$

where $\gamma_{t}, \gamma_{t+1}$ are given in Equation (17). The stochastic Euler equation (26) implies a family of population orthogonality conditions. The GMM estimation method uses these moment conditions to construct an objective function whose optimizers are the estimates of the parameters of the asset pricing model, i.e., $\beta$ and $\Gamma=\left(\Gamma_{1}, \cdots, \Gamma_{\kappa_{\mathcal{S}}}\right)^{\prime}$. The Euler equation in (26) can be written as an $m$ dimensional error term,

$$
h\left(\mathbf{x}_{t+1}, \Gamma, \beta\right)=\beta C_{t}^{\Delta \gamma_{t+1}}\left(c_{t+1}\right)^{-\gamma_{t+1}} \mathbf{R}_{\bullet t+1}-\iota_{m},
$$

where $\mathbf{R}_{\bullet t+1}=\left(R_{1, t+1}, \cdots, R_{m, t+1}\right)^{\prime}$, which measures the deviation from the equilibrium condition. Let $\mathbf{x}_{t+1}=\left(c_{t+1}, \mathbf{R}_{\bullet t+1}^{\prime}\right)^{\prime}$, then the first order condition becomes

$$
E_{t}\left[h\left(\mathbf{x}_{t+1}, \Gamma, \beta\right)\right]=\mathbf{0} .
$$

Given a set $\mathbf{z}_{t}$ of $\varphi$ instruments available at time $t$, a family of population orthogonality conditions can be constructed based on the following moments functions ${ }^{2}$

$$
E\left[h\left(\mathbf{x}_{t+1}, \Gamma_{0}, \beta_{0}\right) \otimes \mathbf{z}_{t}\right]=0 .
$$

Given the conditional moment restrictions (23) and (27), and assuming that the constituents of $h\left(\mathbf{x}_{t+1}, \Gamma_{0}, \beta\right), \mathbf{f}\left(\mathbf{w}_{t}, \alpha\right)$, and the variables in $\mathbf{z}_{t}$ and $\mathbf{w}_{t}$ have finite second moments (Hansen and Singleton (1982a) $)$, we construct a system of unconditional mean equations by combining the unconditional moment equations in system (23) with sys-

\footnotetext{
${ }^{2} h\left(\mathbf{x}_{t+1}, \Gamma_{0}, \beta_{0}\right) \otimes \mathbf{z}_{t}$ stands for the Kronecker product of the $m \times 1$ vector $h\left(\mathbf{x}_{t+1}, \Gamma_{0}, \beta_{0}\right)$ and the $q \times 1$ vector of instruments $\mathbf{z}_{t}$ (including the constant term). The product is an $m q \times 1$ vector.
} 
tem 27). Let $f_{j}\left(\mathbf{w}_{t}, \alpha\right), j=1, \cdots, \kappa_{\mathcal{S}}$,

$$
f_{j}\left(\mathbf{w}_{t}, \alpha\right)=\left(\begin{array}{c}
\mathcal{S}_{t j}-\mathbb{P}_{t}\left(\mathcal{S}_{t j}=1\right) \\
\left(\mathcal{S}_{t j}-\mathbb{P}_{t}\left(\mathcal{S}_{t j}=1\right)\right) \otimes \mathbf{w}_{t}
\end{array}\right)
$$

and $\mathbf{f}\left(\mathbf{w}_{t}, \alpha\right)=\left(f_{1}\left(\mathbf{w}_{t}, \alpha\right)^{\prime}, \cdots, f_{\kappa_{\mathcal{S}}}\left(\mathbf{w}_{t}, \alpha\right)^{\prime}\right)^{\prime} ;$ here $\mathbf{w}_{t}$ is redefined to include a constant term. The system of moment conditions that combines the multinomial logit for the unobserved state variable $\mathcal{S}_{t}$ and the CCAPM Euler equation is

$$
g_{t}\left(\boldsymbol{\Theta}, \mathbf{w}_{t}, \mathbf{x}_{t+1}, \mathbf{z}_{t}\right)=\left(\begin{array}{c}
\mathbf{f}\left(\mathbf{w}_{t}, \alpha\right) \\
h\left(\mathbf{x}_{t+1}, \Theta\right) \otimes \mathbf{z}_{t}
\end{array}\right)
$$

where $\boldsymbol{\Theta}=\left(\Gamma_{1}, \cdots, \Gamma_{\kappa_{\mathcal{S}}}, \beta, \alpha_{1}^{\prime}, \cdots, \alpha_{\kappa_{\mathcal{S}}}^{\prime}\right)^{\prime}$. The unconditional moments generated by the instruments $\mathbf{w}_{t}$ and $\mathbf{z}_{t}$ satisfy

$$
\mathbb{E}\left[g_{t}\left(\mathcal{X}_{t}, \boldsymbol{\Theta}_{0}\right)\right]=0
$$

where $\mathcal{X}_{t}=\left(\mathbf{x}_{t+1}, \mathcal{Z}_{t}\right), \mathcal{Z}_{t}=\left(\mathbf{z}_{t}, \mathbf{w}_{t}\right)$, and the moment function

$$
g_{t}\left(\mathcal{X}_{t}, \boldsymbol{\Theta}_{0}\right)=\zeta\left(\mathbf{y}_{t+1}, \boldsymbol{\Theta}_{0}\right) \otimes \mathcal{Z}_{t}
$$

with $\zeta\left(\mathbf{x}_{t+1}, \boldsymbol{\Theta}_{0}\right)=\left[\left\{\mathcal{S}_{t j}-\mathbb{P}\left(\mathcal{S}_{t j}=1\right)\right\}_{j=1}^{\kappa_{\mathcal{S}}}, h\left(\mathbf{x}_{t+1}, \Theta\right)\right]^{\prime}$.

The number of moment conditions, denoted $\varpi$, in system $(30)$ is $\varpi=\kappa_{\mathcal{S}}(1+$ $\mathcal{K})+m \times \varphi$. The number of unknown parameters in the model $\phi$ is $\kappa_{\mathcal{S}} \mathcal{K}+\kappa_{\mathcal{S}}+1$, representing the $\mathcal{K}$ parameters in each $\alpha_{j}, j=1, \cdots, \kappa_{S}$, the $\kappa_{\mathcal{S}}$ parameters in the expected risk aversion $\gamma_{t}$, and the parameter $\beta$. For the model to be identified, the number of orthogonality conditions $\varpi$ must be at least equal to the number of unknown parameters $\phi$ in the model, implying in this case a counting condition for identification that $m \cdot \varphi \geq 1$.

The GMM estimation uses the sample versions of the population orthogonality 
conditions 30 to construct an estimator for $\Theta_{0}$. The GMM estimator $\widehat{\Theta}$ solves

$$
\min _{\Theta \in \Theta} g_{T}(\mathcal{X}, \Theta)^{\prime} W_{T} g_{T}(\mathcal{X}, \Theta),
$$

where $g_{T}(\mathcal{X}, \Theta)=\frac{1}{T} \sum_{t=1}^{T} g\left(\mathcal{X}_{t}, \Theta\right), W_{T}$ is a sequence of symmetric positive-definite weighting matrices which converge to a positive definite matrix $W$ when $T$ goes to infinity, $\Theta$ is a compact parameter space, $\Theta \subset \mathbb{R}^{\phi}$, and $\mathcal{X}=\left\{\mathcal{X}_{1}, \cdots, \mathcal{X}_{T}\right\}$. Regularity conditions for the consistency of the GMM estimator $\widehat{\Theta}$ in 31 include: (a) $g_{T}(\mathcal{X}, \Theta)$ converges to $\mathbb{E}\left(g\left(\mathcal{X}_{t}, \Theta\right)\right)$ uniformly in $\Theta \in \Theta$, (b) $\mathbb{E}\left(g\left(\mathcal{X}_{t}, \Theta\right)\right) \neq 0$ for all $\Theta \neq \Theta_{0}$, (c) $\mathbb{E}\left(g\left(\mathcal{X}_{t}, \Theta\right)\right)$ and $g_{T}(\mathcal{X}, \Theta)$ are continuously differentiable and, $\frac{\partial g_{T}(\mathcal{X}, \Theta)}{\partial \Theta}$ converges to $\frac{\partial \mathbb{E}\left(g\left(\mathcal{X}_{t}, \Theta\right)\right)}{\partial \Theta}$. If in addition, (d) $\sqrt{T} g_{T}\left(\mathcal{X}, \Theta_{0}\right)$ converges in distribution to a normal distribution with mean zero and variance $V_{g}>0$, and (e) the $\varpi \times \phi$ matrix $G_{0}=$ $\left.\frac{\partial \mathbb{E}\left(g\left(\mathcal{X}_{t}, \Theta\right)\right)}{\partial \Theta^{\prime}}\right|_{\Theta=\Theta_{0}}$ has full rank $\phi$, then $\sqrt{T}\left(\widehat{\Theta}-\Theta_{0}\right)$ converges in distribution to a normal distribution with mean zero and asymptotic variance

$$
\begin{aligned}
\operatorname{Asy} V(\widehat{\Theta}) & =\left(G_{0}^{\prime} W G_{0}\right)^{-1} G_{0}^{\prime} W \Omega_{0} W G_{0}\left(G_{0}^{\prime} W G_{0}\right)^{-1} \\
\Omega_{0} & =E\left[g\left(\mathcal{X}_{t}, \Theta_{0}\right) g_{t}\left(\mathcal{X}_{t}, \Theta_{0}\right)^{\prime}\right] .
\end{aligned}
$$

See Davidson and MacKinnon (2004) and Greene (2008) for a discussion of the asymptotic properties of minimum distance estimators.

Assumption (b) is the global identification which requires that the population moment condition only holds at one parameter value in the entire parameter space $\Theta$. In nonlinear models, it is rarely possible to derive testable conditions for global identification. This assumption is replaced by assumption (e), which is a local identification condition defined in the neighborhood of $\Theta_{0}$. It is also known as the first order identification condition of (Dovonon and Renault, 2008).

The first order conditions for GMM estimation in the SDRA model are a set of $\phi$ 
equations

$$
\frac{\partial \bar{g}_{T}(\mathcal{X}, \Theta)^{\prime}}{\partial \Theta} W_{T} \bar{g}_{T}(\mathcal{X}, \Theta)=0
$$

The first derivatives of the moment function with respect to the parameters

$$
\frac{\partial \bar{g}_{T}(\mathcal{X}, \Theta)}{\partial \Theta}=\left[\frac{\partial \mathbf{f}\left(\mathbf{w}_{t}, \alpha\right)}{\partial \Theta}, \frac{\partial h\left(\mathbf{x}_{t+1}, \beta, \Gamma_{1}, \cdots, \Gamma_{\kappa \mathcal{S}}\right)}{\partial \Theta}\right]^{\prime},
$$

where

$$
\frac{\partial \mathbf{f}\left(\mathbf{w}_{t}, \alpha\right)}{\partial \Theta}=\left[\begin{array}{ccccccc}
0 & 0 & \cdots & 0 & -\frac{\partial \pi_{1 t}}{\partial \alpha_{1}} & \cdots & -\frac{\partial \pi_{1 t}}{\partial \alpha_{\kappa_{\mathcal{S}}}} \\
\cdots & \cdots & \cdots & \cdots & \cdots & \cdots & \cdots \\
0 & 0 & \cdots & 0 & -\frac{\partial \pi_{\kappa_{\mathcal{S}} t}}{\partial \alpha_{1}} & \cdots & -\frac{\partial \pi_{\kappa_{\mathcal{S}}}}{\partial \alpha_{\kappa_{\mathcal{S}}}} \\
\mathbf{0}_{\mathcal{K}} & \mathbf{0}_{\mathcal{K}} & \cdots & \mathbf{0}_{\mathcal{K}} & -\mathbf{w}_{t} \frac{\partial \pi_{1 t}}{\partial \alpha_{1}} & \cdots & -\mathbf{w}_{t} \frac{\partial \pi_{1 t}}{\partial \alpha_{\kappa_{\mathcal{S}}}} \\
\cdots & \cdots & \cdots & \cdots & \cdots & \cdots & \cdots \\
\mathbf{0}_{\mathcal{K}} & \mathbf{0}_{\mathcal{K}} & \cdots & \mathbf{0}_{\mathcal{K}} & -\mathbf{w}_{t} \frac{\partial \pi_{\kappa_{\mathcal{S}} t}}{\partial \alpha_{1}} & \cdots & -\mathbf{w}_{t} \frac{\partial \pi_{\kappa_{\mathcal{S}} t}}{\partial \alpha_{\kappa_{\mathcal{S}}}} \\
& & & & & &
\end{array}\right]
$$

and

$$
\frac{\partial h\left(\mathbf{x}_{t+1}, \beta, \gamma_{1}, \cdots, \gamma_{\mathcal{S}}\right)}{\partial \Theta^{\prime}}=\left[\begin{array}{c}
-\beta c_{t+1}^{-\gamma_{t+1}} C_{t}^{-\Delta \gamma_{t+1}} \log \left(c_{t+1}\right) \mathbf{R}_{\bullet t+1} \otimes \mathbf{z}_{t} \\
-\beta c_{t+1}^{-\gamma_{t+1}} C_{t}^{-\Delta \gamma_{t+1}}\left[\pi_{j, t+1} \log \left(c_{t+1}\right)+\Delta \pi_{j, t+1} \log C_{t}\right] \mathbf{R}_{\bullet t+1} \otimes \mathbf{z}_{t} \\
\cdots \\
\left.-\beta c_{t+1}^{-\gamma_{t+1}} C_{t}^{-\Delta \gamma_{t+1}\left[\pi_{\kappa_{\mathcal{S}}, t+1} \log \left(c_{t+1}\right)+\Delta \pi_{\kappa \mathcal{S}}, t+1\right.} \log C_{t}\right] \mathbf{R}_{\bullet t+1} \otimes \mathbf{z}_{t} \\
c_{t+1}^{-\gamma_{t+1}} C_{t}^{-\Delta \gamma_{t+1}} \mathbf{R}_{\bullet t+1} \otimes \mathbf{z}_{t} \\
\mathbf{0}_{m \cdot \varphi} \\
\mathbf{0}_{m \cdot \varphi} \\
\mathbf{0}_{m \cdot \varphi}
\end{array}\right] .
$$

In the case of the multinomial logit, the first derivative of the conditional mean of $\mathcal{S}_{t}$ 
with respect to the parameters of interest is

$$
\begin{aligned}
& \frac{\partial \pi_{j t}}{\partial \alpha_{j}}=\pi_{j t}\left(1-\pi_{j t}\right) \cdot \mathbf{w}_{t}^{\prime} \text { for } j=1, \cdots, \kappa_{\mathcal{S}} \\
& \frac{\partial \pi_{j t}}{\partial \alpha_{k}}=-\pi_{j t} \pi_{k t} \cdot \mathbf{w}_{t}^{\prime} \text { for } k \neq j=1, \cdots, \kappa_{\mathcal{S}} .
\end{aligned}
$$

There are $\varpi-\phi$ remaining linearly independent moment conditions that are not set to zero in the estimation and must be close to zero if the model is correct. The GMM enables joint estimation and testing of the parameters in the over-identified system of unconditional moments equations when $\varpi>\phi$. The GMM test for validity of the moment conditions is conditional on the ancillary hypotheses about the model specification of $\mathcal{S}_{t}$ and $\mathbf{w}_{t}$. The tests are joint tests of the time-varying specification (including the law of motion of $\mathcal{S}_{t}$ ) and the pricing implications of the CCAPM.

In an over-identified model, there may not be a parameter value $\Theta$ that satisfies (30). The standard test statistic for over-identifying restrictions (also called a $J$ test) is based on the minimized GMM criterion function,

$$
J_{T}(\widehat{\Theta})=T g_{T}(\mathcal{X}, \widehat{\Theta})^{\prime} W_{T} g_{T}(\mathcal{X}, \widehat{\Theta})
$$

When the moment conditions are valid, the $J$ test has an asymptotic $\chi_{\mathrm{df}}^{2}$ with degrees of freedom $\mathrm{df}=\varpi-\phi$. It is worth noting that the $J$ test statistic is a Wald test for the hypothesis, $\mathbb{E}\left(g\left(\mathcal{X}_{t}, \Theta_{0}\right)\right)=0$. The latter is a joint hypothesis with $\varpi$ individual moment restrictions.

\section{Choosing the Weight matrix}

The optimal weight matrix $W_{0}$ which minimizes $(32)$ is $W_{0}=\Omega_{0}^{-1}$. The covariance matrix of the efficient GMM estimator is $\operatorname{Asy} V(\widehat{\Theta})=\left(G_{0}^{\prime} \Omega_{0}^{-1} G_{0}\right)^{-1}$ and is optimal in the class of GMM estimators with this set of moment conditions. In practice, the efficient GMM estimator is unfeasible since $\Omega_{0}^{-1}$ is not known. Hansen (1982) 
shows that a consistent estimator of $\Omega_{0}$ is sufficient for asymptotic efficiency. If $\widetilde{\Theta}$ is a consistent estimator for $\Theta_{0}$, then

$$
\Omega_{T}(\widetilde{\Theta})=\frac{1}{T} \sum_{t=1}^{T} g_{t}\left(\mathcal{X}_{t}, \widetilde{\Theta}\right) g\left(\mathcal{X}_{t}, \widetilde{\Theta}\right)^{\prime},
$$

is a consistent estimator for $\Omega_{0}$. The sample covariance matrix of $g_{t}\left(\mathcal{X}_{t}, \Theta_{0}\right)$ has the form in 35 because the moment functions $\left\{\zeta\left(\mathbf{x}_{t+1}, \Theta\right) \otimes \mathcal{Z}_{t}\right\}_{t=1}^{\infty}$ are martingale first differences. This is a direct implication of the conditional moment restriction (30). With the asset pricing application in mind, this moment condition is consistent with an economy where investors hold assets for one period. The moment condition can be made more general by considering an economy where assets are held to maturity $s>1$

$$
\mathbb{E}\left[\zeta\left(\mathbf{x}_{t+s}, \Theta_{0}\right) \mid \mathfrak{I}_{t}\right]=0, t=1, \ldots, T
$$

The assumption of $s>1$ does not affect the asymptotic properties of the GMM estimators. However, in finite samples, the difficulty in accurately estimating the spectral density matrix (long run variance) of the moment functions is an additional source of poor finite sample performance of the asymptotic approximation. See, for example, Burnside and Eichenbaum (1996). In a general case with $n_{j} \geq 1$, the long run sample variance will be a function of autocovariances up to lag $n_{j}-1$. Notice that the second moments in $(33)$ accounts for a heteroskedastic Euler error term with constant unconditional variance. Hansen (1982) shows that a consistent estimator of $\Omega_{0}$ is sufficient for asymptotic efficiency.

If $\widetilde{\Theta}$ is a consistent estimator for $\Theta_{0}$, then

$$
\Omega_{T}(\widetilde{\Theta})=\frac{1}{T} \sum_{t=1}^{n} g_{t}\left(\mathcal{X}_{t}, \widetilde{\Theta}\right) g_{t}\left(\mathcal{X}_{t}, \widetilde{\Theta}\right)^{\prime}
$$

is a consistent estimator for $\Omega_{0}$. Therefore Hansen's (1982) (feasible) efficient two-step 
GMM estimator $\hat{\Theta}$ solves:

$$
\widehat{\Theta}=\arg \min _{\Theta} \bar{g}_{T}(\Theta)^{\prime} \Omega_{T}(\widetilde{\Theta})^{-1} \bar{g}_{T}(\Theta)
$$

An efficient two-step GMM estimator, denoted $\widehat{\Theta}^{[2]}$, is based on a weight matrix

$$
W_{T}\left(\widehat{\Theta}^{[1]}\right)=\Omega_{T}\left(\widehat{\Theta}^{[1]}\right)^{-1}
$$

where $\widehat{\Theta}^{[1]}$ is a consistent one-step estimator for $\Theta_{0}$ based on a weighting matrix equal to the identity matrix.

The iterative GMM estimator IT-GMM, denoted $\widehat{\Theta}_{i t}$, continues from the two-step estimator by re-estimating the weighting matrix. For each subsequent step $l=3, . ., L$, the weighting matrix is updated using $W_{T}\left(\widehat{\Theta}^{[l-1]}\right)=\Omega_{T}\left(\widehat{\Theta}^{[l-1]}\right)^{-1}$, where $\widehat{\Theta}^{[l-1]}$ is the consistent estimator when $W_{T}=W_{T}\left(\widehat{\Theta}^{(l-2)}\right)$. This is repeated until $l$ attains some large value $L$ (we choose $L=15$ ) or until convergence, defined as $\left\|W_{T}\left(\widehat{\Theta}^{[l+1]}\right)-W_{T}\left(\widehat{\Theta}^{[l]}\right)\right\|<$ $1 E-4$

Instead of taking the weighting matrix as given in each iteration, Hansen et al. (1996) propose an estimator in which the weighting matrix is continuously updated. Formally, the CU-GMM estimator, denoted $\widehat{\Theta}_{c u}$, is

$$
\widehat{\Theta}_{c u}=\arg \min _{\Theta} g_{T}(\mathcal{X}, \Theta)^{\prime} \Omega_{T}(\Theta)^{-1} g_{T}(\mathcal{X}, \Theta)
$$

where $\Omega_{T}(\Theta)=\frac{1}{T} \sum_{t=1}^{T} g\left(\mathcal{X}_{t}, \Theta\right) g\left(\mathcal{X}_{t}, \Theta\right)^{\prime}$. From the point of view of pricing considerations, a weight matrix equal to identity leads to interesting results. The case of $W_{T}=I_{\mathrm{df}}$ corresponds to the so-called first stage GMM. This weight matrix, although not optimal in the econometric sense, does preserve the structure of assets and their economically interesting characteristics. It implies equal weighting of the pricing errors and ignores cross products of pricing errors as well as cross products of the assets' returns. It is equivalent to the traditional least squares approach. Technically, this 
weight matrix allows comparison of pricing errors across models knowing that $\Omega_{T}(\tilde{\Theta})$ did not blow up. This is a computational challenge for the efficient GMM estimation. The squares of small pricing errors are typically also small resulting in large inverse quantities.

Lettau and Ludvigson (2009) studied the properties of the Euler equation errors with the identity weight matrix to preserve the structure of the test assets due to their economical importance. A weight matrix that differs from an identity matrix destroys the model's structure and amounts to minimizing the pricing errors of re-weighted portfolios of the original assets.

In Hansen's 1982) GMM, the weighting matrix $W_{T}^{*}=\Omega_{T}(\widetilde{\Theta})^{-1}$ is optimal in the sense that the estimated parameters have the smallest asymptotic standard errors. In general, the optimal weighting matrix assigns big weights to assets with small variances in their pricing errors, and it assigns small weights to assets with large variances of their pricing errors. The optimal weighting matrix is model dependent and is not suitable for making comparisons among competing models. Hansen and Jagannathan (1997) propose using the sample estimate of $W_{H J}=E\left(\mathbf{R}_{\bullet t} \mathbf{R}_{\bullet t}^{\prime}\right)^{-1}$ as an alternative weighting matrix that is invariant across competing asset pricing models. $W_{H J}$ assigns small weights to assets with big variances and big weights to assets with small variances.

The HJ-distance can only be used for the unconditional pricing moments. Once other available information is added to the conditionning set in the form of instruments $\mathcal{Z}_{t}$, the HJ-distance does not apply.

\section{Asset Market Equilibrium implications}

\section{Pricing errors}

In the standard consumption-based model with CRRA, empirical evidence (Lettau and Ludvigson (2009) ) shows that there are no values of the risk aversion parameter $\gamma$ and the discount factor $\beta$ for which deviations of the Model's Euler equations from the 
equilibrium conditions are not economically large. The pricing error of an asset is the part of the average returns that is not explained by the asset's beta risk.

Let $\xi^{j}$ be the Euler equation errors for the $j$ th asset return defined as:

$$
\begin{array}{r}
\xi_{R}^{j}=\mathbb{E}\left[M_{t+1} R_{j, t+1}\right]-1 \\
\xi_{X}^{j}=\mathbb{E}\left[M_{t+1}\left(R_{j t+1}-R_{f, t+1}\right)\right],
\end{array}
$$

where errors 41) are expressed in terms of excess returns and errors 40 are deviations from the fundamental equilibrium equation (26) (Lettau and Ludvigson, 2009). These equation errors can be interpreted economically as pricing errors defined as the difference between the historical mean excess return and the risk premium implied by the pricing kernel $M_{t+1}$. The risk premium may be written as the product of the asset's beta (exposure) for systematic risk times the price of systematic risk. The pricing error (or the asset's alpha), denoted $\alpha^{j}$, for set $j$ is therefore $\alpha^{j}=\xi_{X}^{j} / \mathbb{E}\left(M_{t+1}\right)$ Lettau and Ludvigson (2009)). Given that the scale factor $\mathbb{E}\left(M_{t+1}\right)^{-1} \approx 1$ (equal to the mean of the risk-free rate), the pricing errors are proportional to Euler equation errors. One testable implication under the null hypothesis that the standard model is true is that both errors should be zero for any traded asset.

\section{Implied risk Premium}

The cyclical properties of the pricing kernel are of interest because they determine the cyclical properties of risk premia. Following Chen et al. (2013), the risk premium $R P_{t}$

$$
R P_{t}=\frac{-\operatorname{Cov}\left(M_{t+1}, R_{\mathrm{M}, t+1}-R_{F, t+1}\right)}{\mathbb{E}\left(M_{t+1}\right)},
$$

where $R_{\mathrm{M}, t+1}$ denotes the return on the market portfolio, for example the CRSP valueweighted stock market index, and $R_{F, t+1}$ denotes the risk-free rate, like the 3-month Treasury-bill rate. 
In the framework of this paper where the risk aversion varies endogenously in the economy but exogenously with respect to the consumers, the pricing kernel in the basic SDRA model we consider is given by:

$$
M_{t+1}=\beta\left(\frac{U^{\prime}\left(C_{t+1}\right)}{U^{\prime}\left(C_{t}\right)}\right)
$$

The assumption that $\gamma_{t}$ is exogenous to the intertemporal choice of the representative consumer is very important. This implies that the consumer does not make intertemporal decisions about $\gamma_{t}$. The risk aversion parameter is endogenous to the economy and is endogenously determined by economy-wide factors that are exogenous to the agents and their preferences.

The marginal utility is dependent on the time-varying state dependent preference parameter:

$$
\begin{aligned}
U^{\prime}\left(C_{t}\right) & =C_{t}^{-\gamma_{t}} \\
M_{t+1} & =\beta \frac{C_{t+1}^{-\gamma_{t+1}}}{C_{t}^{-\gamma_{t}}} .
\end{aligned}
$$

Proposition 5.1. Under the basic SDRA model, the primary testable restriction, $\mathbb{E}_{t}\left[M_{t+1} R_{t+1}\right]=0$, can be linearized and written as:

$$
\begin{aligned}
R P_{t} & =\left[\sum_{j=1}^{\kappa_{\mathcal{S}}} \gamma_{j} \pi_{j, t+1}\right] \operatorname{Cov}_{t}\left(R_{t+1}-R_{F, t+1}, \Delta c_{t+1}\right), \\
& +\log C_{t} \operatorname{Cov}_{t}\left(R_{t+1}-R_{F, t+1}, \Delta \gamma_{t+1}\right) .
\end{aligned}
$$

Equation (43) identifies two sources of risk. The first is the well-understood consumption risk due to covariation between asset returns and changes in nondurables (logarithm) consumption. The novelty in our framework is that this risk varies with the prediction about the state of the economy. The second source of risk is due to changes in the representative agent's level of risk aversion. This is due to the covariance term between the returns and the $\Delta \gamma_{t+1}$. Under SDRA, changes in marginal 
utility are ascribed to changes in risk aversion as well as changes in consumption. The risk premium captures the two sources of risk since both can covary with returns.

The price of consumption risk $\operatorname{Cov}\left(R_{t+1}, \Delta c_{t+1}\right)$ is given by the conditional expectation of the state variable $\mathbb{E}_{t}\left(\gamma_{t+1}\right)$, and the price of the preferences risk, $\operatorname{Cov}\left(R_{t+1}, \Delta \gamma_{t+1}\right)$, is given by the log of consumption. If returns and risk aversion are positively correlated, then the second risk contributes positively to the risk premium, unless $C_{t}<1$.

Holding the risky asset entails a larger risk to marginal utility due to the coincidence of high returns and to lower marginal utility obtained through high consumption and low risk aversion. This second risk can explain some of the equity risk premium puzzle without excessive inflating of the preference parameters.

Gordon and St-Amour (2004) provide a framework that results in similar decomposition of risk along consumption risk and preferences risk. In their Proposition 1, the equity premium is given by

$$
R P_{t}=\gamma_{t} V\left(R_{t}\right)\left(\operatorname{Cov}\left(\tilde{c}_{t}, R_{t+1}\right) / \tilde{c}_{t}\right)+\log \left(\tilde{c}_{t}\right) V\left(R_{t}\right) \operatorname{Cov}\left(\gamma_{t+1}, R_{t+1}\right)
$$

where $\tilde{c}_{t}$ is a scaled consumption level. In addition to the standard source of risk in the constant- $\gamma$ CCAPM ascribed to the covariance of returns with changes in consumption, Equation (44) establishes that changes in the risk aversion $\gamma_{t}$ also contribute to changes in the marginal utility.

Corollary 5.2. The basic SDRA model for the risk premium for any traded asset $i$ has the following decomposition

$$
R P_{i, t}=\underbrace{\left[\frac{\mathbb{E}_{t}\left(\gamma_{t+1}\right) \sigma_{t}^{c c}}{\mathbb{E}_{t}\left(M_{t+1}\right)}\right]}_{\lambda_{t}^{c}} \underbrace{\left(\frac{\sigma_{t}^{\mathcal{R} c}}{\sigma_{t}^{c c}}\right)}_{\beta_{i, t}^{c}}+\underbrace{\left[\frac{\log C_{t} \sigma_{t}^{\gamma \gamma}}{\mathbb{E}_{t}\left(M_{t+1}\right)}\right]}_{\lambda_{t}^{\gamma}} \underbrace{\left(\frac{\sigma_{t}^{\mathcal{R} \gamma}}{\sigma_{t}^{\gamma \gamma}}\right)}_{\beta_{i, t}^{\gamma}}
$$

The parameter $\lambda_{t}^{c}$ measures the price of consumption risk as in the classic CCAPM with constant risk aversion. This risk is the same for all assets. The novel priced risk 
in this SDRA model is $\lambda_{t}^{\gamma}$, which measures the price for aversion risk. This is also the same across all assets. The parameter $\beta_{i, t}^{c}$ (respectively, $\beta_{i, t}^{\gamma}$ ) measures the quantity of consumption (respectively, risk aversion) risk of asset $i$. Given this decomposition, $\lambda_{t}^{c} \beta_{i, t}^{c}$ is the premium for consumption risk, and the new term $\lambda_{t}^{\gamma} \beta_{i, t}^{\gamma}$ is the premium for curvature risk. This paper will investigate whether this additional component of the premium due to curvature can provide some resolution for the risk premium puzzle (Mehra and Prescott, 1985b; Weil, 1989).

\section{Data description}

The empirical analysis is based on the (unconditional) Euler equation errors for the cross-sections of asset returns. These unconditional Euler equations are constructed from the conditional equilibrium conditions using the conditioning information and the instruments $\mathcal{Z}_{t}$.

The cross-section of asset returns includes a broad stock market index return proxied by the Center for Research in Security Prices (CRSP) value-weighted price index return and denoted $R_{M, t}$, a short term Treasury-bill rate measured as the 3 month Treasury Bill rate and denoted $R_{F, t}$, a six size and book-to-market sorted portfolio returns available from Kenneth French's Darthmouth web site, and returns on the 25 size and book-to-market sorted stock portfolios studied by Fama and French (1993).

The equity returns on size and book-to-market sorted portfolios are ideal for empirical tests of asset returns because they exhibit a representative cross-sectional dispersion in expected returns and provide a simple and powerful characterization of the crosssection of average returns.

The sample period is January 1960 through December 2016. Data are collected on three popular predictors of stock returns and are used in the literature as instruments in the GMM estimation (Brandt and Wang, 2003): (1) the default spread (measured as the yield difference between Moody's Baa- and Aaa- rated corporate bonds), and 
the term spread (measured as the yield difference between the 10- and 1-year Treasury bonds).

Consumption in a given month is measured as the end-of-period and the growth of consumption from period $t$ to $t-1$. The measure of real aggregate personal consumption used in this paper is the personal consumption expenditures (in constant 1987 dollars) on nondurables and services (NDS) taken from the United States National Income and Product Accounts. Monthly per capita consumption is obtained by dividing the real aggregate consumption by the total population, including armed forces overseas.

The nominal monthly risk-free rate of interest is the one-month Treasury Bill return from the Center for Research in Security Prices (CRSP) at the University of Chicago. The real risk-free rate is calculated as the nominal risk-free rate, divided by the onemonth inflation rate, based on the deflator defined for nondurables and services (NDS) consumption. As a proxy for the nominal, monthly market return, we take the valueweighted aggregate nominal monthly return (capital gain plus dividends) on all stocks listed on the NYSE and AMEX, obtained from CRSP.

The real monthly market return is calculated as the nominal market return, divided by the one-month inflation rate. The size portfolios are also obtained from CRSP and are formed with all stocks listed on the NYSE and AMEX.

Recession forecasts are complicated by the fact that the state of the economy is an unobserved variable. To that end, this paper takes the recession dates of the business cycle dating committee of the National Bureau of Economic Research (NBER) as a gold-standard chronology of the unobserved state of the economy. The business cycle variable is constructed using the standard NBER dating of the U.S. business cycle. The variables in $\mathbf{w}_{t}$ characterize the state of the economy and include both macroeconomic and financial variables.

Data are collected on variables that have been used in the literature to predict and characterize the business cycle. Estrella and Mishkin (1998) studied and compared the out-of-sample performance of series such as interest rates, spreads, stock prices, 
and monetary aggregates in predicting the U.S. recessions, and find that stock prices as well as some macroeconomic indicators are useful. Nyberg (2008) uses a dynamic probit model to forecast recessions in Germany with variables that include domestic and foreign term spreads, stock market returns, and interest rate differentials between the U.S. and Germany.

In this paper, the potential determinants of changes in risk aversion are variables that pertain to information about the macroeconomic activity in the U.S. These variables consist of 134 time series variables used in Stock and Watson (2002a b, 2006) to forecast inflation and industrial production. This strategy enables use of all information available about the state of the economy. It overcomes the statistical issue of selecting a small subset of variables to represent the information about the economy, especially since the macroeconomic and financial variables tend to be highly correlated. The high dimensional aspect of $\mathbf{w}_{t}$ is addressed using factor models to condense the information in $\mathbf{w}_{t}$ to a small number of factors, also known as diffusion indexes (Stock and Watson, 2002a).

The diffusion index model assumes that $\mathbf{w}_{t}$ admits the following factor model representation with $r$ common latent factors $F_{t}$ :

$$
\mathbf{w}_{t}=\Lambda F_{t}+\xi_{t}
$$

where $F_{t}=\left(f_{1 t}, \cdots, f_{r t}\right)^{\prime}$ are $r$-dimensional stationary processes, $\xi_{t}$ is a $\mathcal{K}$-vector of idiosyncratic disturbances, and $\Lambda$ is a $\mathcal{K} \times r$ matrix of factor loadings. See De Mol et al. (2008) for details on regularity conditions, estimation, and inference about the factors.

The factors $F_{t}$ are unobserved and the number of common factors $r$ is also unknown. There are several methods for determining the number of factors $r$. We follow Bai and $\mathrm{Ng}(2002)$ and use information criteria to penalize the sum of squared residuals in model 45 to construct a consistent estimator for $r$.

Principal components regression is one popular method for computing the common factors. For $\tau=T_{0}, \cdots, T_{1}$, let $\widehat{F}$ denote the $T \times r$ matrix of the first $r$ principal 
components of the predictors $\mathbf{w}$. Let $\mathfrak{I}_{\tau}^{f}=\operatorname{span}\left\{\widehat{f}_{i t}, i=1, \cdots r, t=\tau-T, \cdots, \tau\right\}$ with $r \ll \mathcal{K}$ denoting a parsimonious representation of the information set $\mathfrak{I}_{\tau}$. Consider the spectral decomposition of the sample covariance matrix of $\mathbf{w}, S_{\mathbf{w}}=\mathbf{w}^{\prime} \mathbf{w} /(T-h-p)$ :

$$
S_{\mathbf{w}} V=V D
$$

where $D=\operatorname{diag}\left(d_{1}, \cdots, d_{\mathcal{K}}\right)$ is a diagonal matrix, with $d_{i}$ corresponding to the $i^{\text {th }}$ highest eigenvalue of $S_{\mathbf{w}}$, and $V=\left(v_{1}, \cdots, v_{\mathcal{K}}\right)$ is the matrix whose columns correspond to the normalized eigenvectors of $S_{\mathbf{w}}$. The normalized principal components are defined as :

$$
\widehat{f}_{i, t}=\frac{1}{\sqrt{d_{i}}} v_{i}^{\prime} \mathbf{w} t, \text { for } i=1, \cdots, \mathcal{K}^{*}
$$

where $\mathcal{K}^{*} \leq \mathcal{K}$ is the number of non-zero eigenvalues (De Mol et al., 2008). For the remainder of the empirical analysis, we use the common factors of the 134 macroeconomic variables as indicators of the state of the economy in the modle for $\mathcal{S}_{t}$. Therefore, the $\mathbf{w}_{t}$ is redefined to refer to the estimated common factors $\hat{F}_{t}$ and the number of variables in $\mathbf{w}_{t} \mathcal{K}$ is equivalent to the estimated number of fators $r$. We follow Bai and $\mathrm{Ng}$ (2002), who propose two information criteria that deliver consistent estimators of the number of factors, and we choose the criteria they denote, $I C_{p 2}$. We find an estimated number of factors $\hat{r}=7$ for the cross-section of THE economic and financial time series represented in the 134 variables. As menTioned earlier, this number also represenTS $\mathcal{K}$, the size of $\mathbf{w}_{t}$.

Table 1 summariZes the returns and instruments used in the GMM estimation. Combination E1-Z1 refers to the unconditional Euler equations with two assets $R_{M, t}$ and $R_{F, t}$. This specification is of interest because all asset pricing models seek to match the empirical properties of these two assets. The model is required to price the Treasury Bill and the market portfolio. In this case, the model is exactly identified.

The other specifications require the model to price the returns on the 6 (E2-) and 25 (E3-) portfolios constructed by Fama and French (1993) in which firms are sorted by 
Table 1: Euler equations estimated, $\mathbb{E}_{t}\left(M_{t+1} \mathbf{R}_{\bullet t+1}\right)=\mathbf{1}_{m \cdot \varphi}$, asset returns, instruments $\mathcal{Z}_{t}$ for the GMM estimation

\begin{tabular}{|c|c|c|c|c|c|}
\hline & & \multicolumn{4}{|c|}{ Instruments $\mathcal{Z}_{t}=\left(\mathbf{z}_{t}, \mathbf{w}_{t}\right)$} \\
\hline & & $\mathbf{1}_{T} \hat{F}_{t}$ & $\begin{array}{l}\mathbf{1} \Delta C_{t} \\
\mathbf{R}_{\bullet t} \hat{F}_{t}\end{array}$ & \multicolumn{2}{|l|}{$\mathbf{1} R_{m, t}$ DPrem $_{t}$} \\
\hline \multirow{7}{*}{$\mathbf{R}_{\bullet t+1}$} & $R_{f, t+1} R_{m, t+1}$ & E1-Z1 & E1-Z2 & E1-Z3 & 2 \\
\hline & $R_{f, t+1} R_{m, t+1} 6 F F$ & $\mathrm{E} 2-\mathrm{Z} 1$ & $\mathrm{E} 2-\mathrm{Z} 2$ & E2-Z3 & 8 \\
\hline & $R_{f, t+1} R_{m, t+1} 25 F F$ & E3-Z1 & E3-Z2 & E3-Z3 & 27 \\
\hline & $(\varphi, \mathcal{K}), \mathcal{K} \equiv \hat{r}$ & $(1,7)$ & $(2+m, 7)$ & $(5,7)$ & - \\
\hline & $\phi$ & $8 \kappa_{\mathcal{S}}+1$ & $8 \kappa_{\mathcal{S}}+1$ & $8 \kappa_{\mathcal{S}}+1$ & - \\
\hline & & $8 \kappa_{\mathcal{S}}+m$ & $8 \kappa_{\mathcal{S}}+m \cdot(2+m)$ & $8 \kappa_{\mathcal{S}}+5 m$ & - \\
\hline & $\mathrm{df}=\varpi-\phi$ & $m-1$ & $m \cdot(2+m)-1$ & $5 m-1$ & - \\
\hline
\end{tabular}

Note: Each model represents a combination of returns and instruments which provides a different set of population equations and over-identification conditions. $\varphi$ is the number of instruments, $\mathcal{K}$ is the number of economic indicators which is equal to the number of estimated common factors in $\hat{F}_{t}, \phi$ is the number of parametErs to be estimated in $\Theta, \varpi$ is the total number of estimating equations, and df is the number of over-identifying equations which is equal to the degrees of freedom of the asymptotic $\chi^{2}$ distribution of the J-test.

the market value of their equity (size) and the book-to-market ratio. The information set available to the investors include Z2 and Z3. Note that model specifications (E3-) are highly over-identified. We know that in these cases, tests for over-identification may be less reliable and oversized.

While our task is to estimate equity risk premia in the future, much of the data we use to make these estimates is in the past. Most investors and managers, when asked to estimate risk premia, look at historical data. In fact, the most widely used approach to estimating equity risk premia is the historical premium approach, where the actual returns earned on stocks over a long time period is estimated and compared to the actual returns earned on a default risk-free rate (usually government security). The difference, on an annual basis, between the two returns is computed and represents the historical risk premium.

While users of risk and return models may have developed a consensus that historical premium is, in fact, the best estimate of the risk premium looking forward, there are surprisingly large differences in the actual premia we observe being used in practice, with the numbers ranging from $3 \%$ at the lower end to $12 \%$ at the upper end on an annual basis. In our analysis, we will estimate the implied annualized risk premia for our models and compare it with the range of values that is observed in the literature. 


\section{$7 \quad$ Empirical Results}

\subsection{Probabilities of recession is a data rich environment}

Our model for the state-dependent risk aversion estimates the state of the economy using infomation in $\mathbf{w}_{t}$. We choose not to restrict our list of variables in $\mathbf{w}_{t}$ and use factor models and principal component analysis to condense all the available information about the economy. We use these high-dimensional dense methods to capture the state of the economy and, in particular the likelihood of periods of recession and expansion. This in itself is a novelty in the literature of characterizing the buisiness cycle and turning points. Most of the existing literature has focussed on key economic indicators such as gross domestic product. For example, Nalewaik (2012) estimates Markov switching models and shows that the growth rate of gross domestic income, deflated by the gross domestic product deflator, has done a better job recognizing the start of recessions than has the growth rate of real GDP. See also, Chauvet and Hamilton (2006), Hamilton (2011), Hamilton (1989), Hamilton (1989).

Estimating states of recession is important because the NBER dating is only available ex-post and not in real-time. To predict future states, it is important to have a model that can accurately indicate recession periods in the future. In this paper, we use real-time information about the economy and perform a pseudo-out-of-sample evaluation of the accuracy of estimated recession probabilities. As mentioned earlier, the information available to the researcher is data on the 134 economic variables pertaining to information about the macroeconomy and financial markets. At each time period, $t$, we extract and estimate the common factors from this cross-section of time series. These $\hat{r}=7$ estimated factors $\hat{F}_{t}$ are then used to estimate the probability of the state variable $\mathcal{S}_{t}=j$ or equally that $\mathcal{S}_{t j}=1$, for $j=1, \cdots, \kappa_{\mathcal{S}}$. We consider the case of two states, $\kappa_{\mathcal{S}}=2$, with $\mathcal{S}_{t 1}=1$ a state of recession and 0 otherwise, $\mathcal{S}_{t 2}=1$ a state of expansion. We also consider $\kappa_{\mathcal{S}}=4$, with $\mathcal{S}_{t j}=1$ when observation corresponds to a 
contraction, $\mathcal{S}_{t 2}=1$ when the observation corresponds to a contraction and is an actual trough date, and similarly $\mathcal{S}_{t 3}=1$ when observation is a state of expansion and $\mathcal{S}_{t 4}=1$ in a state of expansion and is an actual peak. In any case, we have $\sum_{j=1}^{\kappa_{\mathcal{S}}} \mathcal{S}_{t=j}=1$.

Figure (1) represents the estimated probabilities for the state $\mathcal{S}_{1, t}=1$, equivalent to $\mathcal{S}_{t}=1$, for two cases of state dependent definitions of $\mathcal{S}$, one with two states $\kappa_{\mathcal{S}}=2$, in which case recession is represented by one and only one state $j=1$, and these are shown in panels (a) to (c), and the case of 4 states $\kappa_{\mathcal{S}}=4$, in which case one can differentiate between a state of recession $j=1$ and a state of recession and a trough $j=2$, and these results are depicted in panels (d) to (f). For the sake of brevity, the figure shows a representative selection of model specifications in Table (1). Detailed probability estimates for the full set of specifications are available upon request. In general, we observe that during periods that the NBER classifies as a recession, the probability of a recession is high and close to one. These probabilities rise substantially when the NBER recession starts, remaining high until the end of the recession period.

One important observation is that the 4 states model for $\mathcal{S}$ is better at identifying the trough periods. The values of $\hat{\pi}_{t, 2}$ are practically equal to zero outside of the trough periods, which are identified within the NBER recession periods. The probabilities of an obeservation being a recession but not a trough are small, mostly less than $30 \%$ outside of the NBER recession periods.

The proposed big data model for dating the business cycle has the advantage of being readily available to be estimated in real time, whereas the NBER dating is only available ex-post, and generally with long delays. Since one of our goals is to forecast business cycle episodes of recession in real time, we use the information available to the researcher or policy maker in a pseudo-out-of-sample forecasting exercise and use the expost NBER dating as benchmarks for evaluating the real-time forecasting performances of the model. The results suggest that placing focusing on economy-wide information may be useful in assessing the current state of the economy in real time. 
Figure 1: Estimated (monthly) probabilities for the recession state, $\hat{\pi}_{1, t}=$ $\hat{\mathbb{E}}_{t}\left[S_{1, t}=1\right]$ in the case of $\kappa_{\mathcal{S}}=2$, and the recession and through states $\hat{\pi}_{1, t}, \hat{\pi}_{2, t}$, in the case of $\kappa_{\mathcal{S}}=4$, for the state-dependent preferences model.

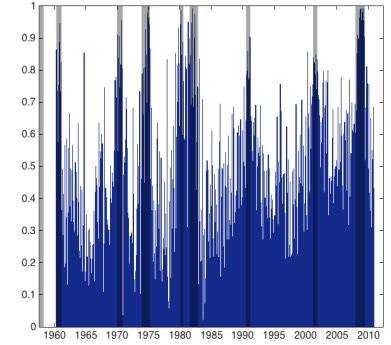

(a) $\kappa_{\mathcal{S}}=2,$, E1-Z1

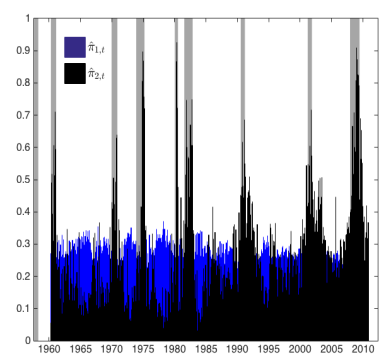

(d) $\kappa_{\mathcal{S}}=4, \mathrm{E} 1-\mathrm{Z} 1$

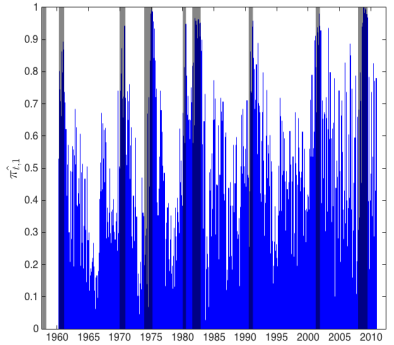

(b) $\kappa_{\mathcal{S}}=2, \mathrm{E} 2-\mathrm{Z} 1$

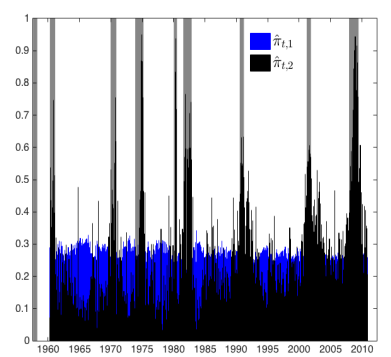

(e) $\kappa_{\mathcal{S}}=4, E 3-Z 1$

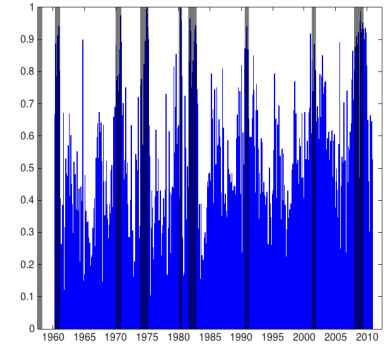

(c) $\kappa_{\mathcal{S}}=2, \mathrm{E} 3-\mathrm{Z} 1$

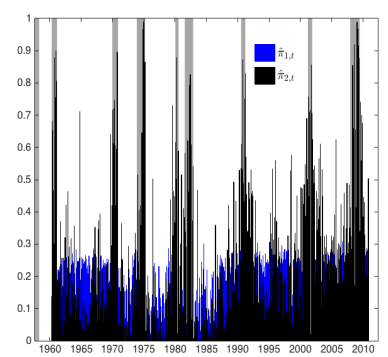

(f) $\kappa_{\mathcal{S}}=4, \mathrm{E} 2-\mathrm{Z} 1$

\subsection{Evidence for counter-cyclical risk aversion}

In this section, we discuss the empirical estimates of the risk aversion parametr $\gamma$ in the (constant) CCAPM model, the parameter $\Gamma$ and the state-dependent preference parameter $\gamma_{t}$ in the SDRA model. The results for the standard CCAPM are presented in Table 2. The parameters $\gamma$ and $\beta$ solve the GMM problem based on the equilibrium Euler equations in (26) and using instruments and returns defined in Table (1). The estimated values of $\beta$ are consistent across the model specifications and are somewhere in the vicinity of one. This is consistent with what is generally observed in this literature. There are no real surprises for the parameter $\gamma$. For the model to fit the equilibrium conditions, the model implies extremly high values of risk aversion. It is well documented in the literature that to be able to fit the CCAPM model, unrealistically high values of $\gamma$ are required. See for example, Tauchen (1986) and Kocherlakota (1990). 
Table 2: Euler equation errors and GMM estimates of the preference parameters

\begin{tabular}{|c|c|c|c|c|c|c|c|c|c|}
\hline & E1-Z1 & $\mathrm{E} 2-\mathrm{Z1}$ & E3-Z1 & E1-Z2 & $\mathrm{E} 2-\mathrm{Z} 2$ & E3-Z2 & E1-Z3 & E2-Z3 & E3-Z3 \\
\hline & \multicolumn{9}{|c|}{ Panel A1: CRRA-I } \\
\hline$\gamma$ & 99.51 & 21.99 & 19.09 & 17.76 & 15.13 & 10.14 & 14.03 & 5.17 & 13.47 \\
\hline$\beta$ & 0.9489 & 1.014 & 1.011 & 1.080 & 0.997 & 1.105 & 0.991 & 0.992 & 1.035 \\
\hline \multirow[t]{2}{*}{ Rmse } & 0.098 & 0.037 & 0.044 & 0.091 & 0.016 & 0.012 & 0.058 & 0.082 & 0.035 \\
\hline & \multicolumn{9}{|c|}{ Panel A2: CRRA-HJ } \\
\hline$\gamma$ & 99.54 & 100.30 & 70.94 & 30.56 & 90.5 & 72.18 & 10.45 & 8.00 & 5.81 \\
\hline$\beta$ & 0.9488 & 0.947 & 1.006 & 1.141 & 1.365 & 1.308 & 0.993 & 0.998 & 1.00 \\
\hline Rmse & 0.066 & 0.051 & 0.069 & 0.093 & 0.054 & 0.0297 & 0.055 & 0.013 & 0.030 \\
\hline
\end{tabular}

Notes: The table reports estimated values of the preference parameters $\hat{\beta}$ and $\hat{\gamma}$ chosen to minimize the mean square pricing error in Equation 26 for different sets of returns and instruments.

Table 3: Euler equation errors and GMM estimates of the preference parameters

\begin{tabular}{|c|c|c|c|c|c|c|c|c|c|}
\hline \multirow[b]{3}{*}{ Rmse } & E1-Z1 & E1-Z2 & E1-Z3 & E2-Z1 & E2-Z2 & E2-Z3 & E3-Z1 & E3-Z2 & E3-Z3 \\
\hline & \multicolumn{9}{|c|}{ Panel A1- $I: \kappa_{\mathcal{S}}=2$} \\
\hline & 0.012 & 0.014 & 0.007 & 0.0147 & 0.010 & 0.0146 & 0.089 & 0.058 & 0.025 \\
\hline$\beta$ & 0.990 & 0.974 & 0.939 & 0.974 & 0.970 & 0.931 & 0.990 & 0.990 & 0.982 \\
\hline \multicolumn{10}{|l|}{$\gamma_{t}$} \\
\hline Mean & 0.152 & 0.195 & 1.355 & 0.195 & 0.373 & 0.310 & 0.299 & 0.309 & 0.339 \\
\hline Std.dev & 0.005 & 0.815 & 1.162 & 0.815 & 0.100 & 0.574 & 0.0009 & 0.0017 & 0.0019 \\
\hline$\Gamma_{1}$ & 0.499 & 0.476 & 0.309 & 0.476 & 0.489 & 0.331 & 0.439 & 0.399 & 0.339 \\
\hline \multirow[t]{2}{*}{$\Gamma_{2}$} & 0.343 & 0.348 & 0.309 & 0.348 & 0.410 & 0.290 & 0.339 & 0.309 & 0.239 \\
\hline & \multicolumn{9}{|c|}{ Panel A1-HJ: $\kappa_{\mathcal{S}}=2$} \\
\hline Rmse & 0.091 & 0.021 & 0.023 & 0.096 & 0.010 & 0.010 & 0.078 & 0.015 & 0.025 \\
\hline $\bar{\beta}$ & 0.991 & 0.991 & 1.012 & 0.991 & 0.995 & 0.999 & 0.998 & 0.991 & 0.982 \\
\hline \multicolumn{10}{|l|}{$\gamma_{t}$} \\
\hline Mean & 0.499 & 0.311 & 0.499 & 0.311 & 0.324 & 0.300 & 0.342 & 0.309 & 0.361 \\
\hline Std.dev & 0.0004 & 0.591 & 0.551 & 0.591 & 0.013 & 0.002 & 0.0003 & 0.0017 & 0.0002 \\
\hline$\Gamma_{1}$ & 0.503 & 0.584 & 0.499 & 0.504 & 0.455 & 0.296 & 0.341 & 0.308 & 0.354 \\
\hline$\Gamma_{2}$ & 0.419 & 0.398 & 0.500 & 0.418 & 0.205 & 0.304 & 0.342 & 0.308 & 0.367 \\
\hline
\end{tabular}

Notes: The table reports estimated values of the preference parameters $\hat{\beta}$ and $\hat{\Gamma}$ chosen to minimize the mean square pricing error in Equation 26 for different sets of returns and instrument. The root-mean-squared-error $\%$ Rmse is the pricing error computed as a proportion of the average squared returns of assets. We show results for weighting matrices equal to identity, $W=I$, and equal to Hansen-Jaganathan, $W=\mathbb{E}\left(\mathbf{R}^{\prime} \mathbf{R}\right)^{-1}$.

The estimated model fits the data well in terms of $\%$ Rmse, the errors in sample pricing equations relative to the size of average returns. These conclusions are invariant to the choice of the weighting matrix.

Table (3) reports our estimation results for the structural parameters $(\beta, \Gamma)$ in the the state-dependent preferences model defined by a two state variable $\mathcal{S}_{t}$ with $\kappa_{\mathcal{S}}=2$, the unconditional moments equations in (30), and by the specifications of returns and instruments as in Table (1). Panel A1-I corresponds to a weighting matrix $W_{T}$ in (34) equal to the identity matrix, and Panel A1-HJ corresponds to the special case where the weight matrix is equal to Hansen-Jaganathan's $W_{T}=\mathbb{E}\left(\mathbf{R}_{\bullet} t \mathbf{R}_{\bullet t}^{\prime}\right)^{-1}$. The estimated values for the discount factor $\beta$ are as expected and within the values found 
Table 4: Correlation between $\gamma_{t}$ and a leading indicator of the U.S. business cycle

\begin{tabular}{|c|c|c|c|c|c|c|c|c|c|}
\hline & E1-Z1 & E1-Z2 & E1-Z3 & E2-Z1 & E2-Z2 & E2-Z3 & E3-Z1 & E3-Z2 & E3-Z3 \\
\hline & & -0.613 & -0.671 & $\begin{array}{l}-0.609 \\
\end{array}$ & -0.665 & -0.630 & -0.672 & -0.639 & \\
\hline
\end{tabular}

Notes: The table reports sample correlation coefficient corr values between the estimated time series of risk aversion parameter $\gamma_{t}$ and the leading indicator of U.S. business cycle. The results correspond to an identity weight matrix, $W_{T}=I$, and $\kappa_{\mathcal{S}}=2$

Figure 2: The leading indicator of the United States economic activity and business cycle.

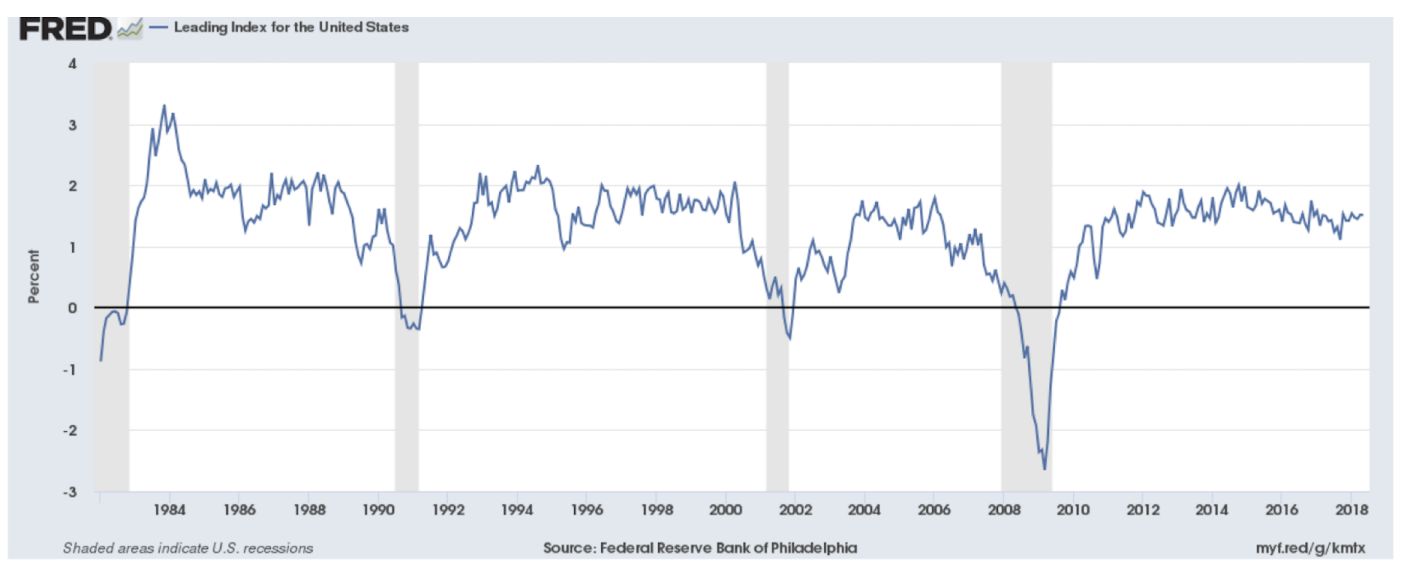

in the literature and in the constant CCAPM model estimated earlier. The mean values of the estimated state-dependent $\gamma_{t}$ seem reasonable especially with the low reported sample standard errors. The ranges of the estimated $\gamma_{t}$ appear to agree with results in the literature on fixed risk aversion. Mehra and Prescott (1985a) argue that a risk aversion in the range of 10 and below seems reasonable. Epstein and Zin (1991), in their recursive utility model, estimate a risk aversion between 0.14 and 1.14 . Kim (2014) uses a non-parametric framework where $\gamma_{t}$ is a smooth function of time and finds average values of risk aversion in the range $[0.276,2.015]$ for a model calibrated with an elasticity of intertemporal substitution equal to 1.5. The SDRA model points to a representative agent with a level of risk aversion that is neither excessive nor highly volatile.

The estimates of the $\Gamma$ parameter show that there is asymmetry in the risk preferences of the representative agent depending on the state of the economy. For example, the estimated values of $\Gamma_{1}$, which is the component of risk aversion associated with $\mathcal{S}_{t}$ which is a state of recession, are generally higher than the estimated values of $\Gamma_{2}$. This 
is true for both choices of weight matrix. There is an excess risk aversion, $\Gamma_{1}-\Gamma_{2}$, associated with the economy being in a bust state. This counter-cyclical risk aversion may suggest that market participants are susceptible to booms and busts. Furthermore, fear may be an important factor that drives this counter-cyclicality. Agents experiencing higher levels of fear in the bust state predict a significantly lower investment in the risky asset. This has been recognized previously in the literature. In traditional CCAPM literature, the habit formation model (Campbell and Cochrane, 1999a; Constantinides, 1990) provides one of the leading explanations for the cyclical behavior of risk aversion. The level of habit and consumption together form the surplus-consumption ratio. Fluctuations of risk aversion are linked to the business cycle by interpreting a boom state to a state in which the level of consumption is well above habit. Cohn et al. (2015) report experimental evidence, with financial professionals, that risk eversion is counter-cyclical, and a causal relationship between fear and risk preferences. Generally, empirical support from financial studies is at best inconclusive.

To the best of our knowledge, this is the first study which modifies a widely used asset pricing model such that the risk aversion is explicitly allowed to vary with information about the state of the economy. We do not pursue the same stategy as in Kim (2014) who models the aversion parameter as a function of time. We use information available from the wide macroeconomy and financial markets to model the risk preferences of consumers. Table (4) reports sample estimates of the correlation coefficient between the estimated time-varying risk aversion parameter $\hat{\gamma}_{t}$ and a leading indicator of the U.S. business cycle. The leading index predicts the six-month growth rate of the Coincident Economic Activity Index $3^{3}$ of the United States (seasonally adjusted, index July $1992=100$ ). Figure (2) plots the coincident indicator and the NBER recession dates. The sample correlations are within the range $[-59 \% ;-67 \%]$, showing positive co-movement between the estimated risk aversion and the fluctuations in the coincident indicator. The latter is a good indicator of the business cycle and is pro-cyclical. The

\footnotetext{
${ }^{3}$ Source: Federal Reserve Bank of St. Louis FRED
} 
Figure 3: SDRA estimates of risk aversion, $\hat{\gamma}_{t}$, for model specification E2-Z1

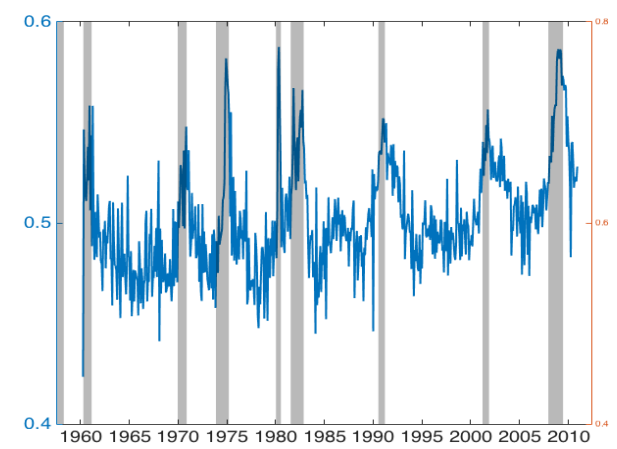

(a) $\kappa_{\mathcal{S}}=2, W_{T}=I$

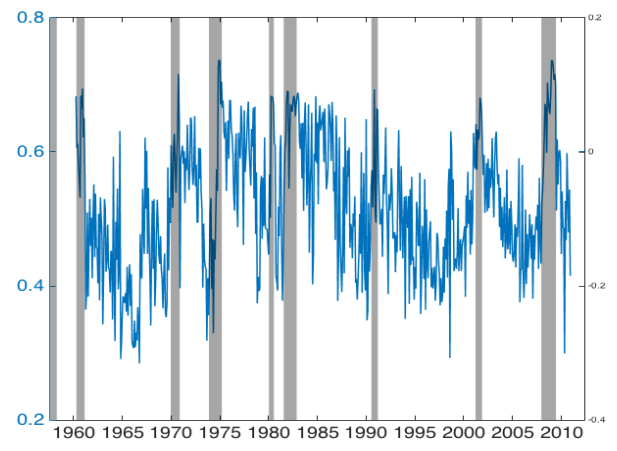

(c) $\kappa_{\mathcal{S}}=4, W_{T}=I$

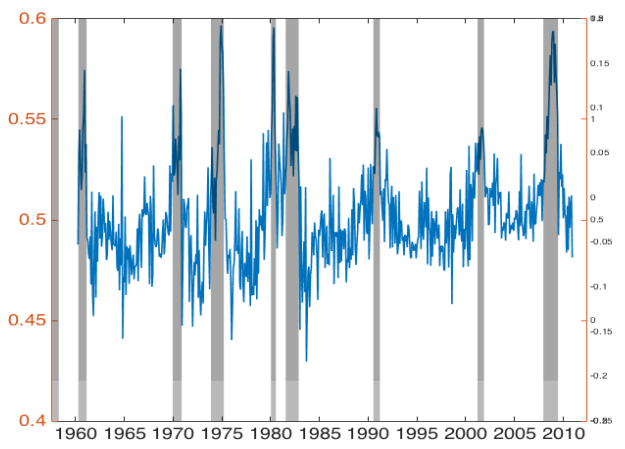

(b) $\kappa_{\mathcal{S}}=2, W_{T}=E\left(\mathbf{R}_{\bullet} \mathbf{R}_{\bullet}^{\prime}\right)^{-1}$

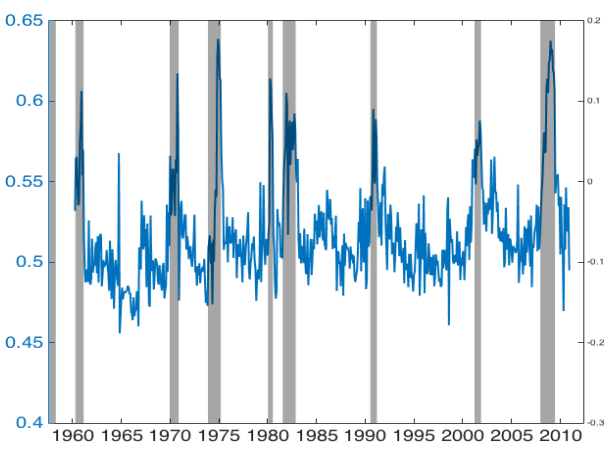

(d) $\kappa_{\mathcal{S}}=4, W_{T}=E\left(\mathbf{R}_{\bullet} \mathbf{R}_{\bullet t}^{\prime}\right)^{-1}$

estimated risk aversion indicates that consumers become more risk averse with negative growth rate in the coincident indicator, that is, when the economy is in a condition of recession.

Figure (3) plots the estimated $\hat{\gamma}_{t}$ for a selection of the models considered in Table (1). In all panels, the fluctuations in $\hat{\gamma}_{t}$ (shown in blue) are counter-cyclical. There is an increase in risk aversion in the last recession that followed the 2008 financial crisis compared to the previous recessions. Furthermore, the time variation is also apparent in the spread of the estimated risk aversion. In Figure (4), we observe from the fluctuations in the percentage change in the estimated risk aversion $\left(\log \left(\hat{\gamma}_{t} / \hat{\gamma}_{t-1}\right) \times 100\right)$ an increased volatility during times of economic hardship, especially during the early 1980 recession and the recent 2008 financial crisis. This is a new finding that shows a phenomenon of "volatility boom" in risk aversion during recession times. This evidence 
Figure 4: Fluctuations of changes in estimated risk aversion, $\log \left(\gamma_{t} / \gamma_{t-1}\right)$ in percent, case of the SDRA Model E2-Z1

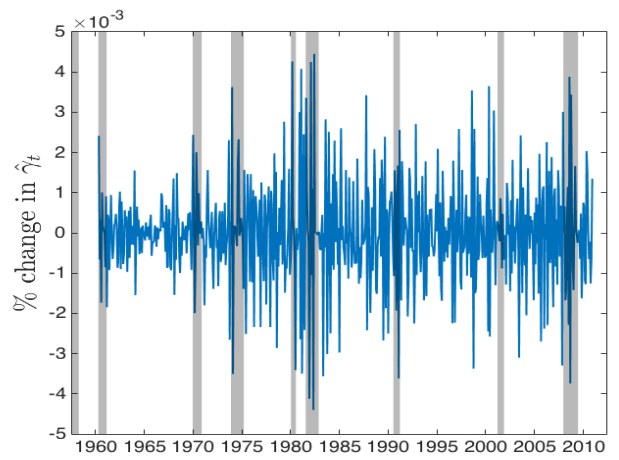

(a) $\kappa_{\mathcal{S}}=2, W_{T}=I_{\mathrm{df}}$

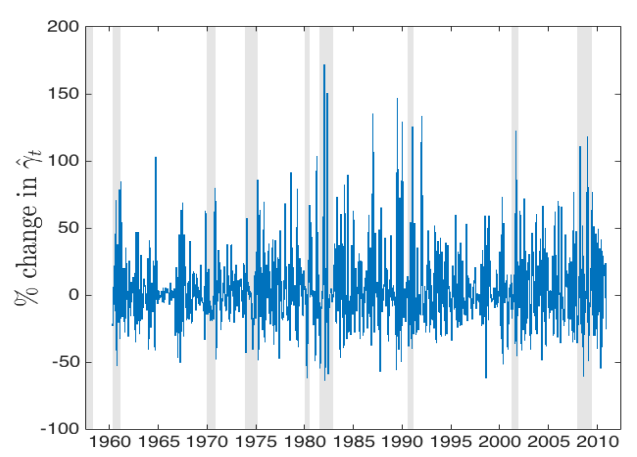

(b) $\kappa_{\mathcal{S}}=2, W_{T}=E\left(\mathbf{R}_{\bullet t} \mathbf{R}_{\bullet}^{\prime}\right)^{-1}$

Table 5: Monthly estimated Implied Risk Premium

\begin{tabular}{|c|c|c|c|c|c|c|c|c|c|}
\hline & E1-Z1 & E2-Z1 & E3-Z1 & E1-Z2 & $\mathrm{E} 2-\mathrm{Z2}$ & E3-Z2 & E1-Z3 & E2-Z3 & E3-Z3 \\
\hline & \multicolumn{9}{|c|}{ Monthly $R P_{t} \%$} \\
\hline CCAPM & 0.0800 & 0.0266 & 0.0211 & 0.0545 & 0.0046 & 0.0013 & 0.0043 & 0.0015 & 0.0414 \\
\hline SDRA $\kappa_{\mathcal{S}}=2$ & 0.1344 & 0.0908 & 0.0937 & 0.0959 & 0.0943 & 0.0953 & 0.1202 & 0.1025 & 0.1048 \\
\hline$C_{t}$ Risk: $\lambda_{t}^{c} \beta_{i, t}^{c}$ & 0.1150 & 0.0850 & 0.0920 & 0.0943 & 0.0940 & 0.0951 & 0.0909 & 0.0946 & 0.1042 \\
\hline$\gamma_{t}$ Risk: $\lambda_{t}^{\gamma} \beta_{i, t}^{\gamma}$ & 0.0194 & 0.0058 & 0.0017 & 0.0016 & 0.0003 & 0.0001 & 0.0293 & 0.0079 & 0.0006 \\
\hline
\end{tabular}

Notes. Implied risk premium is computed as in Equation (30). The premia are monthly and in percent. The per annum premium can be obtained by compounding the monthly premia. The GMM estimation is with weighting matrix equal to identity.

reinforces the notion that consumers' preferences can be an important business cyle indicator. This finding is also consistent with the evidence that stock volatility increases during recessions and financial crises, which can be linked to many factors (Schwert (1989)), some of which can be ascribed to fluctuations in the consumers' changes in their attitudes towards risk.

\subsection{Implications for Asset Returns}

\section{Implied Risk Premium}

Under Proposition (5.1), unless $\gamma_{t}$ is constant and the model is a standard CCAPM and/or the consumption level is equal to unity, changes in marginal utility are ascribed to changes in $\gamma_{t}$ as well as to changes in consumption growth. The risk premium 
Table 6: Euler equation pricing errors

\begin{tabular}{|c|c|c|c|c|c|c|c|c|c|c|}
\hline \multirow{2}{*}{$\begin{array}{l}\text { Size } \\
\text { Quintile }\end{array}$} & \multicolumn{10}{|c|}{ Book-to-market Equity (BE/ME) Quintiles } \\
\hline & Low & BM2 & BM3 & $\overline{\mathrm{BM} 4}$ & High & Low & BM2 & BM3 & BM4 & High \\
\hline & \multirow{2}{*}{\multicolumn{10}{|c|}{$\begin{array}{cc}\text { Panel A. Constant CCAPM Model E3-Z1 } \\
\text { Pricing Errors (\%) } & \text { Standard Errors }(\%)\end{array}$}} \\
\hline & & & & & & & & & & \\
\hline Small & 1.7703 & 1.7774 & 1.7836 & 1.7946 & 1.7919 & 0.3219 & 0.2764 & 0.2392 & 0.2271 & 0.2418 \\
\hline ME2 & 1.7748 & 1.7806 & 1.7785 & 1.7799 & 1.7900 & 0.2880 & 0.2401 & 0.2153 & 0.2088 & 0.2420 \\
\hline ME3 & 1.7754 & 1.7823 & 1.7764 & 1.7848 & 1.7861 & 0.2662 & 0.2171 & 0.1981 & 0.1953 & 0.2245 \\
\hline ME4 & 1.7850 & 1.7866 & 1.7739 & 1.7810 & 1.7773 & 0.2378 & 0.2039 & 0.1975 & 0.1925 & 0.2284 \\
\hline \multirow[t]{3}{*}{ Big } & 1.7899 & 1.7857 & 1.7945 & 1.7802 & 1.8018 & 0.1879 & 0.1787 & 0.1719 & 0.1873 & 0.2093 \\
\hline & \multirow{2}{*}{\multicolumn{5}{|c|}{$\begin{array}{l}\text { Panel B. Time V } \\
\text { Pricing Errors (\%) }\end{array}$}} & -2 & $\kappa_{S}=2$, & $\bar{V}=I_{\mathrm{df}}$ & & \\
\hline & & & & & & \multicolumn{5}{|c|}{ Standard Errors (\%) } \\
\hline Small & 0.3243 & 0.2784 & 0.2409 & 0.2288 & 0.2436 & 0.3219 & 0.2764 & 0.2392 & 0.2271 & 0.2418 \\
\hline ME2 & 0.2901 & 0.2419 & 0.2168 & 0.2104 & 0.2438 & 0.2880 & 0.2401 & 0.2153 & 0.2088 & 0.2420 \\
\hline ME3 & 0.2682 & 0.2187 & 0.1996 & 0.1967 & 0.2262 & 0.2662 & 0.2171 & 0.1981 & 0.1953 & 0.2245 \\
\hline ME4 & 0.2396 & 0.2054 & 0.1990 & 0.1939 & 0.2301 & 0.2378 & 0.2039 & 0.1975 & 0.1925 & 0.2284 \\
\hline Big & 0.1893 & 0.1800 & 0.1732 & 0.1887 & 0.2109 & 0.1879 & 0.1787 & 0.1719 & 0.1873 & 0.2093 \\
\hline
\end{tabular}

Note: The reported numbers are computed as the sample values of the pricing errors, $\xi_{R}^{j}$, the pricing errors in the Euler equations. See equation 40 .

captures both sources of risk because the returns covary with both consumption growth and changes in $\gamma_{t}$. In this section, we estimate the mean of the implied preference risk $\lambda_{t}^{\gamma} \beta_{i, t}^{\gamma}$ and of the implied consumption risk $\lambda_{t}^{c} \beta_{i, t}^{c}$. This feature of the SDRA model can potentially explain the observed risk premium with reasonable levels of risk aversion.

The equity market risk premium is the average return that investors require over the risk-free rate for accepting the higher variability in returns that are common for equity investments. As the name implies, this rate should not take into account any risk factors. The risk-free rate is commonly approximated by reference to the yield on long-term debt instruments issued by presumably financially healthy governments (e.g. AAA-rated government bonds with a maturity of 30 years).

Table (5) presents the sample mean of the risk premium implied by the CCAPM as well as the means of the components of the implied risk premium as predicted by the SDRA model. Note that in our data, we compute a monthly historical risk premium of $0.27 \%$ (equivalent to $3.4 \% 4$ per annum).

The historical equity premium is more than double the implied premium in the SDRA model and three times that of the CCAPM. The implied price for curvature

\footnotetext{
${ }^{4}$ Historical long run average of spread between the S\&P 500 and the 10 Year U.S. Treasury Bond.
} 
Table 7: Euler equation pricing errors

\begin{tabular}{|c|c|c|c|c|c|c|c|c|c|c|}
\hline \multirow{2}{*}{$\begin{array}{l}\text { Size } \\
\text { Quintile }\end{array}$} & \multicolumn{10}{|c|}{ Book-to-market Equity (BE/ME) Quintiles } \\
\hline & Low & BM2 & BM3 & BM4 & High & Low & BM2 & BM3 & BM4 & High \\
\hline & \multicolumn{10}{|c|}{ Panel A. Constant CCAPM Model E3-Z1 } \\
\hline & \multicolumn{5}{|c|}{ Pricing Errors (\%) } & \multicolumn{5}{|c|}{ Standard Errors (\%) } \\
\hline Small & -0.4847 & 0.0260 & 0.0879 & 0.3437 & 0.4124 & 1.770 & 1.777 & 1.783 & 1.794 & 1.791 \\
\hline ME2 & -0.3214 & 0.0501 & 0.1622 & 0.2360 & 0.3303 & 1.774 & 1.780 & 1.778 & 1.779 & 1.790 \\
\hline ME3 & -0.2347 & 0.0893 & 0.0415 & 0.2007 & 0.3543 & 1.775 & 1.782 & 1.776 & 1.784 & 1.786 \\
\hline ME4 & -0.0986 & -0.0748 & -0.0250 & 0.1686 & 0.0889 & 1.785 & 1.786 & 1.773 & 1.781 & 1.777 \\
\hline \multirow[t]{3}{*}{ Big } & -0.1881 & -0.1499 & -0.0735 & -0.2370 & 0.1057 & 1.789 & 1.785 & 1.794 & 1.780 & 1.801 \\
\hline & \multirow{2}{*}{\multicolumn{10}{|c|}{ Panel B. Time-varying CCAPM E3-Z1 $\kappa_{S}=4, W=I_{\mathrm{df}}$}} \\
\hline & & & & & & & & & & \\
\hline Small & -0.2880 & 0.1724 & 0.1981 & 0.4282 & 0.5379 & 0.322 & 0.276 & 0.239 & 0.227 & 0.242 \\
\hline ME2 & -0.1680 & 0.1636 & 0.2880 & 0.3602 & 0.4478 & 0.288 & 0.240 & 0.215 & 0.209 & 0.242 \\
\hline ME3 & -0.1024 & 0.2023 & 0.1470 & 0.3008 & 0.4605 & 0.266 & 0.217 & 0.198 & 0.195 & 0.224 \\
\hline ME4 & -0.0076 & 0.0075 & 0.1307 & 0.2832 & 0.2283 & 0.238 & 0.204 & 0.197 & 0.192 & 0.228 \\
\hline Big & -0.1409 & -0.0713 & -0.0242 & -0.1039 & 0.0973 & 0.188 & 0.178 & 0.172 & 0.187 & 0.209 \\
\hline
\end{tabular}

Note: The reported numbers are computed as the sample values of the pricing errors, $\xi_{R}^{j}$, the pricing errors in the Euler equations. See equation 40 .

risk is not high enough to explain the gap between the price of consumption risk and the historical equity premium. The findings may be indicative of a low beta for the curvature risk for the market portfolio (S\&P500). Although the SDRA model explains only $55 \%$ of the equity premium puzzle, it does so without inflating the level of risk aversion. It is worth noting that historical premium values can vary greatly depending on the market portfolio and on the risk-free rate, as well as the method of calculation. A survey of 2394 reported numbers for the risk premia for the U.S. indicates a range between $2.5 \%$ and $15.8 \%$ with an average of $5.7 \%$ (Fernandez et al. (2013)).

\section{Pricing Errors}

In this section we focus on Euler equations which represent the theoretical restrictions from which all asset pricing implications follow. There is large evidence in the literature that tests for conditional Euler equations lead to rejections of the standard CCAPM even with inflated values of the risk aversion parameter Hansen, 1982, Hansen et al. 2008). We study here the unconditional Euler equations, associated with the returns in 1. and compare the CCAPM with the SDRA model. These empirical tests place additional testable restrictions on asset pricing models: not only must these models 
Table 8: Euler equation pricing errors

\begin{tabular}{|c|c|c|c|c|c|c|c|c|c|c|}
\hline \multirow{2}{*}{$\begin{array}{l}\text { Size } \\
\text { Quintile }\end{array}$} & \multicolumn{10}{|c|}{ Book-to-market Equity (BE/ME) Quintiles } \\
\hline & Low & BM2 & BM3 & BM4 & High & Low & BM2 & BM3 & BM4 & High \\
\hline & \multicolumn{10}{|c|}{ Panel A. Constant CCAPM Model E3-Z2 } \\
\hline & \multicolumn{5}{|c|}{ Pricing Errors (\%) } & \multicolumn{5}{|c|}{ Standard Errors (\%) } \\
\hline Small & -0.1486 & -0.7094 & -4.8125 & -0.6927 & -0.4515 & 0.2174 & 0.0109 & 0.0816 & 0.0172 & 0.3220 \\
\hline ME2 & -3.8943 & -0.1963 & 0.0077 & 0.4032 & 0.2921 & 2.5858 & 0.3698 & 0.2765 & 2.2031 & 0.3198 \\
\hline ME3 & 0.0332 & 0.7686 & 0.3294 & 0.2628 & 2.3814 & 0.2392 & 1.9100 & 0.3007 & 0.2272 & 1.7921 \\
\hline ME4 & 0.4729 & 0.3726 & 3.0951 & 0.5931 & -0.3321 & 0.2910 & 0.2419 & 1.8881 & 0.3210 & 0.2881 \\
\hline \multirow[t]{3}{*}{ Big } & -2.4304 & -0.0609 & -0.0014 & 0.4121 & 0.2983 & 2.3680 & 0.3342 & 0.2402 & 1.9484 & 0.2944 \\
\hline & \multirow{2}{*}{\multicolumn{10}{|c|}{$\begin{array}{l}\text { Panel B. Time-varying CCAPM E3-Z2 } \kappa=2, W=I_{\mathrm{df}} \\
\text { Pricing Errors (\%) }\end{array}$}} \\
\hline & & \multicolumn{2}{|c|}{ Pricing Errors (\%) } & & & & & \multicolumn{3}{|c|}{ Standard Errors (\%) } \\
\hline Small & -0.1391 & -0.6899 & -4.8304 & -0.6792 & -0.4336 & 0.2171 & 0.0109 & 0.0813 & 0.0180 & 0.3218 \\
\hline ME2 & -3.9236 & -0.1853 & 0.0262 & 0.3777 & 0.3037 & 2.5842 & 0.3693 & 0.2763 & 2.2012 & 0.3194 \\
\hline ME3 & 0.0519 & 0.7435 & 0.3411 & 0.2817 & 2.3572 & 0.2390 & 1.9081 & 0.3002 & 0.2270 & 1.7903 \\
\hline ME4 & 0.4847 & 0.3912 & 3.0690 & 0.6048 & -0.3138 & 0.2906 & 0.2417 & 1.8863 & 0.3206 & 0.2878 \\
\hline Big & -2.4553 & -0.0492 & 0.0175 & 0.3890 & 0.3105 & 2.3663 & 0.3338 & 0.2400 & 1.9466 & 0.2939 \\
\hline
\end{tabular}

Note: The reported numbers are computed as the sample values of the pricing errors, $\xi_{R}^{j}$, the pricing errors in the Euler equations. See Equation 40 .

place zero pricing errors when the pricing kernel $M_{t+1}$ is correctly specified according to the model, but they must also produce large pricing errors when the pricing kernel is poorly specified, even when the parameters $\gamma, \Gamma, \beta$ are chosen to minimize those errors.

For the results in this section we use the identity matrix, $W_{T}=I$, to weight the GMM criterion function. As mentioned earlier, this weight matrix preserves the structure of the test assets and the Euler equation errors also represent the pricing errors. We study the unconditional Euler equation errors (based on excess returns) for the cross-section of returns defined in Table (1).

Tables (2-3) report the square root of the average squared Euler equation errors (Rmse) as a measure of the magnitude of mispricing,

$$
\begin{aligned}
\text { Rmse } & =\frac{R M S E}{R M S R} \\
R M S E & =\sqrt{\frac{1}{N} g_{T}(\mathcal{X}, \hat{\Theta})^{\prime} g_{T}(\mathcal{X}, \hat{\Theta})} \\
R M S R & =\sqrt{\frac{1}{N} \sum_{j=1}^{N} \mathbf{R}_{j}^{\prime} \mathbf{R}_{j},}
\end{aligned}
$$

where $\mathbf{R}_{j}$ is a $T$ - vector of observations of returns on asset $j$. For the CCAPM model, the lowest Rmse is when the square root of the average pricing errors $R M S E$ is $1.2 \%$ 
the square root of the average squared returns on the 25 size and book-to-market portfolios. This result occurs at a value for risk aversion of $\gamma=10$. For the SDRA model, the smallest Rmse gives a mispricing of $1.0 \%$ the square-root of the average squared returns. These results occur for a value of (monthly) state-dependent risk aversion, $\hat{\gamma}_{t}$, with mean 0.373 and standard errors 0.100 .

Tables (6-8) and Figure (5) present the sample values of the pricing errors, $\xi_{R}^{j}$ in equation (40) and their standard errors, reported for the 25 size and book-to-market sorted portfolio returns available from Kenneth French's Dartmouth web site. These returns are value weighted portfolio returns of common stock sorted into 5 size (market equity) quintiles and five book value-market value quintiles. The results are reported for the intersections of the five size quintiles and the five book-to-market quintiles. The CCAPM pricing errors are generally much larger than those in the SDRA model. In Table 6 for example, the mispricing in the SDRA model ranges from $9 \%$ of the CCAPM mispricing for the quintile portfolio Big-BM3 to $18 \%$ for the quintile portfolio Small-Low.

We also note that the fit of the model depends on the information set used in consructing the unconditional Euler equation, i.e., the choice of the instrumets $\mathbf{Z}$. In Table (8), Euler equation errors with the instruments $\mathbf{Z}_{3}$ for both the CCAPM and the SDRA model are increased compared to $\mathbf{Z}_{1}$. There is also a large variation between the errors in the cross-section of the returns. For the CCAPM, the mispricing ranges from $-3.89 \%$ to $0.007 \%$, while for the SDRA model, this range is $(-3.92 \%, 0.017 \%)$. In the E3-Z2 specification, the instruments set includes the cross-section of the 25 portfolio returns $\mathbf{R}_{\bullet}$, the estimated common factors $\hat{F}_{t}$, and consumption growth. The model in this case is extremly over-identified with degrees of freedom equal to $m \cdot(2+m)-1=$ 782. In the case of GMM with many instruments (possibly increasing more than the sample size), Han and Phillips (2006) report that the usual convergence behavior of the GMM estimator depends on the relative strength of the signal variability compared to the main signal. This quantity is determined by the sample size, the number of 
Figure 5: Euler pricing errors for the individual 25-Fama \& French size and BM portfolios in the SDRA Model E3-Z1

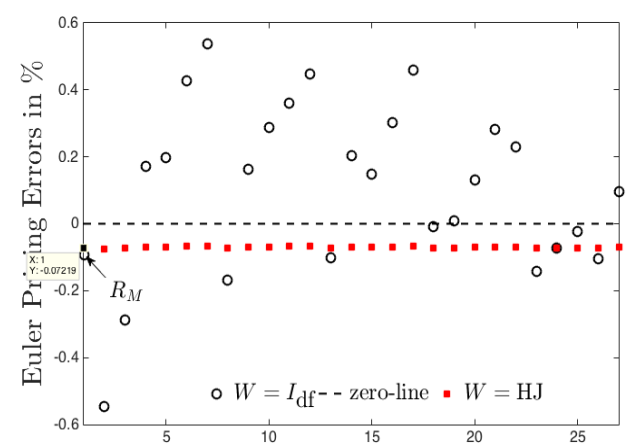

(a) $\kappa_{\mathcal{S}}=4$

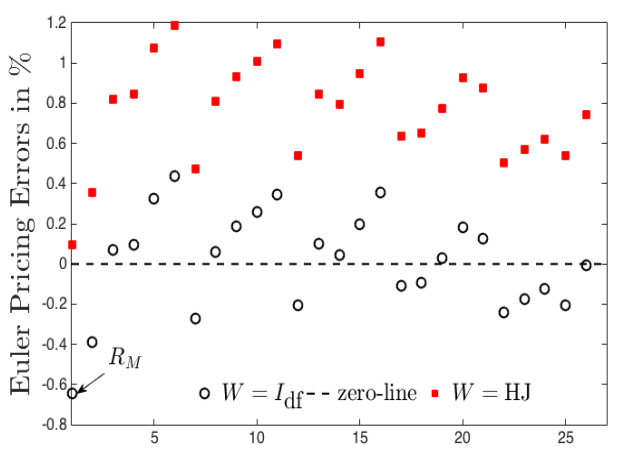

(b) $\kappa_{\mathcal{S}}=2$

moment conditions, and the order of magnitude of the main signal. The quality of information the instruments add to the system must compensate for the increased degrees of freedom. Some of these aspects are also related to the problem of weak instruments known in this literature.

In Figure (5), we plot the pricing errors for each of the 25 quintiles portfolio, in addition to the mispricing associated with a CRSP stock market return. We note that the pricing error for the market portfolio $R_{M}$ is negative for both weight specifications $W_{T}=I$ and $W_{T}=\mathbb{E}\left(\mathbf{R}_{\bullet t} \mathbf{R}_{\bullet t}^{\prime}\right)$, with $-0.072 \%$ and $-0.62 \%$ respectively, implying a negative alpha in the expected return-beta representation of the model for $R_{M}$. This says that unconditional risk premia are too low to be explained by the stock markets' covariance with the systematic risk in the market. This is somehow in contrast with what is found in the previous literature. but given that this model is severely overidentified, inference in this model is problematic.

\section{Conclusion}

We formulate a consumption-based asset pricing model in which aggregate risk aversion is time-varying in response not only to news about aggregate consumption as in the habit formation model, but also to news about a wide range of key economic indicators. 
We model the dynamics of aggregate risk aversion in a more general farmework for modeling fluctuations in aggregate preferences towards risk. We interpret the frameowrk as a reduced form model for an economy where agents fear states of economic bust.

The estimated ranges of risk aversion are realistic, and the estimated time-variation in the preferences is a good indicator of the business cycle. Our empirical results show evidence of counter-cyclical aggregate risk aversion consistent with findings in the literature of counter-cyclical risk premia. Our model describes an economy where changes in the market behavior induced by boom and bust scenarios can be attributed to changes in the consumers' risk preferences. We find evidence that the volatility clustering of the state-dependent risk aversion parameter is counter-cyclical, which may produce feedback loops that amplify market trends. Factors that may reinforce this amplification mechanism include social projection bias where people have a tendency to project their own risk preferences onto others (Cohn et al., 2015). However, unlike (Cohn et al., 2015), we provide an alternative source of risk preferences' changes other than consumers' and/or investors' fear, and a model that links the changes in the aggregate risk preferences to information about the wider economy.

\section{References}

Abel, A. B. (1990). Asset prices under habit formation and catching up with the joneses. The American Economic Review 80(2), 38-42.

Agresti, A. (2012). Categorical data analysis. New Jersey: Wiley.

Bai, J. and S. Ng (2002). Determining the number of factors in approximate factor models. Econometrica 70, 191-221.

Brandt, M. W. and K. Q. Wang (2003). Time-varying Risk Aversion and Unexpected Inflation. Journal of Monetary Economics 50, 1457-1498. 
Breeden, D., M. Gibbons, and R. Litzenberger (1989). Empirical tets of the consumption-Oriented CAPM. Journal of Finance 44, 231-262.

Breeden, D., R. Litzenberger, and T. Jia (2014). Consumption-based asset pricing: Research and applications. Unpublished Manuscript.

Brunnermeier, M. K. and S. Nagel (2008). Do Wealth Fluctuations Generate Timevarying Risk Aversion? Micro-Evidence on Individuals's Asset Allocation. American Economic Review 98, 713-736.

Burnside, C. and M. Eichenbaum (1996). Small sample properties of GMM-based Wald tests. Journal of Business \& Economic Statistics 14(3), 294-308.

Campbell, J. (2003). Consumption-based asset pricing, Volume 1B, pp. 801-885. Elsevier: Amsterdam. in George M. Constantinides, Milton Harris, and René M. Stulz, ed.: Handbook of the Economics of Finance.

Campbell, J. and J. Cochrane (1999a). By force of habit: a consumption-based explanation of aggregate stock market behavior. Journal of Political Economy 10\%, $205-251$.

Campbell, J. Y. (1996). Understanding rosk and return. Journal of Political Economy 104, 298-345.

Campbell, J. Y. and J. H. Cochrane (1999b). By Force of Habit: A Consumption-based Explanation of Aggregate Stock Market Behavior. Journal of Political Economy 10\%, $205-251$.

Chauvet, M. and J. Hamilton (2006). Dating Business Cycle Turning Points, pp. 1-54. San Diego, CA : Elsevier Press.

Chen, X., J. Favilukis, and S. C. Ludvigson (2013). An estimation of economic models with recursive preferences. Quantitative Economics 4, 39-83. 
Cochrane, J. H. (1996). A cross-sectional test of an investment-based asset pricing model. Journal of Political Economy $104(3), 572-621$.

Cohn, A., J. Engelmann, E. Fehr, and M. A. Maréchal (2015). Evidence for countercyclical risk aversion: An experiment with financial professionals. American Economic Review 105(2), 860-885.

Constantinides, G. M. (1990). Habit formation: A resolution of the equity premium puzzle. The Journal of Political Economy 98(3), 519-543.

Davidson, R. and J. MacKinnon (2004). Econometric theory and methods. New York: Oxford University Press.

De Mol, C., D. Giannone, and L. Reichlin (2008). Forecasting using a large number of predictors: Is Bayesian shrinkage a valid alternative to principal components? Journal of Econometrics 146, 318-328.

Dovonon, P. and E. Renault (2008). GMM overidentification test with first order underidentification. Unpublished Manuscript www4.ncsu.edu/dpellet/tec/renault.pdf.

Epstein, L. G. and S. E. Zin (1991). Substitution, risk aversion, and the temporal behavior of consumption and asset returns: An empirical analysis. Journal of Political Economy 99(2), 263-286.

Estrella, A. and F. Mishkin (1998). Predicting u.s. Recessions: Financial Variables as Leading Indicators. Review of Economics and Statistics 80(1), 45-61.

Fama, E. and K. French (1993). Common risk factors in the returns on stocks and bonds. Journal of Financial Economics 33, 3--56.

Fernandez, P., j. Aguirreamalloa, and P. Linares (2013). Market risk premium and risk free rate used for 51 countries in 2013: a survey with 6,237 answers.

Gordon, S. and P. St-Amour (2000). A preference regime model of bear and bull markets. American Economic Review 90. 
Gordon, S. and P. St-Amour (2004). Asset returns and state-dependent risk preferences. Journal of Business and Economic Statistics 22(3), 241-252.

Greene, W. H. (2008). Econometric analysis. Sixth Edition, Pearson Prentice Hall New Jersey.

Hamilton, J. D. (1989). A new approach to the economic analysis of nonstationary time series and the business cycle. Econometrica $5 \%$

Hamilton, J. D. (2011). Calling recessions in real time. International Journal of Forecasting 27, 1006-1026.

Han, C. and P. Phillips (2006, 01). GMM with many moment conditions. Econometrica 74, $147-192$.

Hansen, L. and R. Jagannathan (1997). Assessing specification errors in stochastic discount factor models. Journal of Finance 52, 557-590.

Hansen, L. P. (1982). Large Sample Properties of Generalized Method of Moments Estimators. Econometrica 50(4), 1029-1054.

Hansen, L. P., J. Heaton, and A. Yaron (1996). Finite Sample Properties of Some Alternative GMM Estimators. Journal of Business and Economic Statistics 14(3), $262-280$.

Hansen, L. P., J. C. Heaton, and N. Li (2008). Consumption Strikes Back? measuring Long-Run Risk. Journal of Political Economy 116, 260-302.

Hansen, L. P. and K. Singleton (1982a). Generalized Instrumental Variables Estimation of Nonlinear Rational Expectations Models. Econometrica 50(5), 1269-1286.

Hansen, L. P. and K. Singleton (1982b). Generalized Instrumental Variables Estimation of Nonlinear Rational Expectations Models. Econometrica 50(5), 1269-1286. 
Hansen, L. P. and K. J. Singleton (1983). Stochastic consumption, risk aversion, and the temporal behavior of asset returns. Journal of Political Economy 91(2), 249-265.

Heaton, J. (1995). An empirical investigation of asset pricing with temporally dependent preference specifications. Econometrica 63(3), 681-717.

Kim, K. H. (2014). Counter-cyclical risk aversion. Journal of Empirical Finance 29, $384-401$.

Kocherlakota, R., N. (1990). On tests of representative consumer asset pricing models. Journal of Monetary Economics 26, 285-304.

Lettau, M. and S. Ludvigson (2001). Resurrecting the (C)CAPM: a cross-sectional test when risk premia are time-varying. Journal of Political Economy 109, 1238--1287.

Lettau, M. and S. Ludvigson (2009). Euler equation errors. Review of Economic Dynamics, 255-283.

Maio, P. (2013). Intertemporal capm with conditioning variables. Management Science 59(1), 122-141.

Mankiw, G. and M. Shapiro (1986). Risk and retuen: Consumption beta versus market beta. Review of Economics and Statistics 68(3), 452-459.

Mehra, R. and E. Prescott (1985a). The equity premium: A puzzle. Journal of Monetary Economics 15, 145-61.

Mehra, R. and E. Prescott (1985b). The equity puzzle. Journal of Monetary Economics $15,145-161$.

Melino, A. and A. X. Yang (2003). State-dependent preferences can explain the equity premium puzzle. Review of Economic Dynamics 6, 806-830.

Merton, R. (1973). An intertemporal capital asset pricing model. Econometrica 41, 867-887. 
Nalewaik, J. J. (2012). Estimating probabilities of recession in real time using GDP and GDI. Journal of Money, Credit and Banking 44(1), 235-253.

Nyberg, H. (2008). DynamicProbit Models and Financial Variables in Recession Forecasting. Hesinki Center for Economic Research, discussion paper No. 225.

Schwert, G. W. (1989). Business cycles, financial crises, and stock volatility. NBER Working Paper No. w2957.

Shiller, R. J. (1997). Why Do People Dislike Inflation?, pp. 13-65. Romer, C. D. and Romer, D. H. (eds), Chicago and London: University Chicago Press. in Reducing Inflation: Motivation and Strategy.

Stock, J. H. and M. . Watson (2006). Forecasting with many predictors. In Handbook of Economic Forecasting 1, 551-554.

Stock, J. H. and M. W. Watson (2002a). Forecasting using principal components from a large number of predictors. Journal of the American Statistical Association 97, $1167-1179$

Stock, J. H. and M. W. Watson (2002b). Macroeconomic forecasting using diffusion indexes. Journal of Business and Economic Statistics 20(2), 147-162.

Tauchen, G. (1986). Statistical properties of generalized method-of-moments estimators of structural parameters obtained from financial market data. Journal of Business 83 Economic Statistics 4(4), 397-416.

Weil, P. (1989). The equity premium puzzle and the risk free rate puzzle. Journal of Monetary Economics 24, 401-421. 


\section{APPENDIX A}

\subsection{Proof of Proposition 1}

In basic SDRA model, the stochastic discount factor is given by:

$$
\begin{aligned}
M_{t+1} & =\beta \frac{C_{t+1}^{-\gamma_{t+1}}}{C_{t}^{-\gamma_{t}}} \\
& =e^{\left[\log \beta-\gamma_{t+1} \log C_{t+1}+\gamma_{t} \log C_{t}\right]} \\
& =e^{\log \beta-\gamma_{t+1}\left(\log C_{t+1}-\log C_{t}\right)-\log C_{t} \Delta \gamma_{t+1}} \\
& =e^{\log \beta-\gamma_{t+1} \Delta \log C_{t+1}-\log C_{t} \Delta \gamma_{t+1}}
\end{aligned}
$$

Using the approximation $e^{x} \cong 1+x$ for small $x$, we can write:

$$
M_{t+1} \cong 1+\log \beta-\gamma_{t+1} \Delta \log C_{t+1}-\log C_{t} \Delta \gamma_{t+1}
$$

Now taking the testable imlication of the model after de-meaning all variables,

$$
\begin{aligned}
\mathbb{E}_{t}\left[\left(R_{t+1}-R_{F, t+1}\right) M_{t+1}\right] & =\mathbb{E}_{t}\left[R_{t+1}-R_{F, t+1}\right]-\mathbb{E}_{t}\left[\left(R_{t+1}-R_{F, t+1}\right) \gamma_{t+1} \Delta \log C_{t+1}\right] \\
& -\log C_{t} \mathbb{E}_{t}\left[\left(R_{t+1}-R_{F, t+1}\right) \Delta \gamma_{t+1}\right]=0 \\
\mathbb{E}_{t}\left[R_{t+1}\right]-R_{F, t+1} & =\mathbb{E}_{t}\left[\left(R_{t+1}-R_{F, t+1}\right) \gamma_{t+1} \Delta \log C_{t+1}\right]+\log C_{t} \mathbb{E}_{t}\left[\left(R_{t+1}-R_{F, t+1}\right) \Delta \gamma_{t+1}\right]
\end{aligned}
$$

The random variable $\gamma_{t+1}$ has a distribution that depends on the discrete random variable $S_{t+1}$ which characterizes the state of the economomy. With $\kappa_{\mathcal{S}}$ states, this variable has an expectation equal to $E_{t}\left[\gamma_{t+1}\right]=\sum_{j=1}^{\kappa_{\mathcal{S}}} \gamma_{j} P\left[S_{j, t+1} \mid \mathbf{w}_{t}=1\right]$. Using the 
Law of Iterated Expectations, we can then write the expression in (46) as:

$$
\begin{aligned}
\mathbb{E}_{t}\left[R_{t+1}\right]-R_{F, t+1} & =\sum_{j=1}^{\kappa_{\mathcal{S}}} \gamma_{j} P\left[S_{j, t+1} \mid \mathbf{w}_{t}=1\right] \mathbb{E}_{t}\left[\left(R_{t+1}-R_{F, t+1}\right) \Delta \log C_{t+1}\right] \\
& +\log C_{t} \mathbb{E}_{t}\left[\left(R_{t+1}-R_{F, t+1}\right) \Delta \gamma_{t+1}\right] \\
& =\sum_{j=1}^{\kappa_{\mathcal{S}}} \gamma_{j} P\left[S_{j, t+1} \mid \mathbf{w}_{t}=1\right] \operatorname{Cov}_{t}\left[R_{t+1}-R_{F, t+1}, \Delta \log C_{t+1}\right] \\
& +\log C_{t} \operatorname{Cov}_{t}\left[R_{t+1}-R_{F, t+1}, \Delta \gamma_{t+1}\right] .
\end{aligned}
$$

In addition, given the result that $\mathbb{E}\left(M_{t+1}\right) \approx 1$, this leads to the expression of the proposition:

$$
\begin{aligned}
R P_{t} & =\left[\sum_{j=1}^{\kappa_{\mathcal{S}}} \gamma_{j} \pi_{j, t+1}\right] \operatorname{Cov}_{t}\left[R_{t+1}-R_{F, t+1}, \Delta \log C_{t+1}\right] \\
& +\log C_{t} \operatorname{Cov}_{t}\left[R_{t+1}-R_{F, t+1}, \Delta \gamma_{t+1}\right]
\end{aligned}
$$

Let's use the notation $\sigma_{t}^{\mathcal{R} c}$ to denote $\operatorname{Cov}_{t}\left[R_{t+1}-R_{F, t+1}, \Delta \log C_{t+1}\right]$, and $\sigma_{t}^{\mathcal{R} \gamma}$ to denote $\operatorname{Cov}_{t}\left[R_{t+1}-R_{F, t+1}, \Delta \gamma_{t+1}\right]$. Similarly, let us denote the conditional variance for consumption $\operatorname{Var}_{t}\left(\Delta C_{t+1}\right)$ as $\sigma_{t}^{c c}$. We can rewrite the decomposition of the risk premia, given the approximation $\mathbb{E}_{t}\left(M_{t+1}\right) \approx 1$, into:

$$
R P_{t}=\underbrace{\left[\frac{\mathbb{E}_{t}\left(\gamma_{t+1}\right) \sigma_{t}^{c c}}{\mathbb{E}_{t}\left(M_{t+1}\right)}\right]}_{\lambda_{t}^{c}} \underbrace{\left(\frac{\sigma_{t}^{\mathcal{R} c}}{\sigma_{t}^{c c}}\right)}_{\beta_{i, t}^{c}}+\underbrace{\left[\frac{\log C_{t} \sigma_{t}^{\gamma \gamma}}{\mathbb{E}_{t}\left(M_{t+1}\right)}\right]}_{\lambda_{t}^{\gamma}} \underbrace{\left(\frac{\sigma_{t}^{\mathcal{R} \gamma}}{\sigma_{t}^{\gamma \gamma}}\right)}_{\beta_{i, t}^{\gamma}}
$$

